\title{
The nature of the low-frequency emission of M 51
}

\section{First observations of a nearby galaxy with LOFAR ${ }^{\star}$}

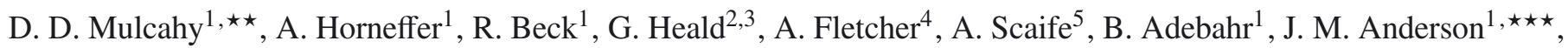
A. Bonafede ${ }^{6}$, M. Brüggen ${ }^{6}$, G. Brunetti ${ }^{7}$, K. T. Chyży ${ }^{8}$, J. Conway ${ }^{9}$, R.-J. Dettmar ${ }^{10}$, T. Enßlin ${ }^{11}$, M. Haverkorn ${ }^{12,13}$, C. Horellou ${ }^{9}$, M. Iacobelli ${ }^{2,13}$, F. P. Israel ${ }^{13}$, H. Junklewitz ${ }^{14}$, W. Jurusik ${ }^{8}$, J. Köhler ${ }^{1}$, M. Kuniyoshi ${ }^{1}$, E. Orrú ${ }^{2}$, R. Paladino ${ }^{15,7}$, R. Pizzo ${ }^{2}$, W. Reich ${ }^{1}$, and H. J. A. Röttgering ${ }^{13}$

${ }^{1}$ Max-Planck-Institut für Radioastronomie, Auf dem Hügel 69, 53121 Bonn, Germany e-mail: d.d.mulcahy@soton.ac.uk

2 ASTRON, Postbus 2, 7990 AA, Dwingeloo, The Netherlands

3 Kapteyn Astronomical Institute, Postbus 800, 9700 AV Groningen, The Netherlands

${ }^{4}$ School of Mathematics and Statistics, Newcastle University, Newcastle-upon-Tyne NE1 7RU, UK

5 School of Physics and Astronomy, University of Southampton, Highfield, SO17 1SJ, Southampton, UK

${ }^{6}$ Universität Hamburg Sternwarte, Gojenbergsweg 112, 21029 Hamburg, Germany

7 INAF-IRA Bologna, via Gobetti 101, 40129 Bologna, Italy

8 Astronomical Observatory, Jagiellonian University, ul. Orla 171, 30-244 Kraków, Poland

9 Dept. of Earth and Space Sciences, Chalmers University of Technology, Onsala Space Observatory, 43992 Onsala, Sweden

${ }^{0}$ Ruhr-Universität Bochum, Astronomisches Institut, 44780 Bochum, Germany

11 Max-Planck-Institut für Astrophysik, Karl-Schwarzschild-Str. 1, 85748 Garching, Germany

12 Department of Astrophysics/IMAPP, Radboud University Nijmegen, PO Box 9010, 6500 GL Nijmegen, The Netherlands

13 Leiden Observatory, Leiden University, PO Box 9513, 2300 RA Leiden, The Netherlands

14 Argelander-Institut für Astronomie, Radio Astronomy Department, Auf dem Hügel 71, 53121 Bonn, Germany

15 Department of Physics and Astronomy, University of Bologna, V.le Berti Pichat 6/2, 40127 Bologna, Italy

Received 12 May 2014 / Accepted 1 July 2014

\section{ABSTRACT}

Context. Low-frequency radio continuum observations $(<300 \mathrm{MHz})$ can provide valuable information on the propagation of low-energy cosmic ray electrons (CRE). Nearby spiral galaxies have hardly been studied in this frequency range because of the technical challenges of low-frequency radio interferometry. This is now changing with the start of operations of LOFAR.

Aims. We aim to study the propagation of low-energy CRE in the interarm regions and the extended disk of the nearly face-on spiral galaxy Messier 51. We also search for polarisation in M 51 and other extragalactic sources in the field.

Methods. The grand-design spiral galaxy M 51 was observed with the LOFAR High Frequency Antennas (HBA) and imaged in total intensity and polarisation. This observation covered the frequencies between $115 \mathrm{MHz}$ and $175 \mathrm{MHz}$ with 244 subbands of 8 channels each, resulting in 1952 channels. This allowed us to use RM synthesis to search for polarisation.

Results. We produced an image of total emission of M 51 at the mean frequency of $151 \mathrm{MHz}$ with $20^{\prime \prime}$ resolution and $0.3 \mathrm{mJy}$ rms noise, which is the most sensitive image of a galaxy at frequencies below $300 \mathrm{MHz}$ so far. The integrated spectrum of total radio emission is described well by a power law, while flat spectral indices in the central region indicate thermal absorption. We observe that the disk extends out to $16 \mathrm{kpc}$ and see a break in the radial profile near the optical radius of the disk. The radial scale lengths in the inner and outer disks are greater at $151 \mathrm{MHz}$, and the break is smoother at $151 \mathrm{MHz}$ than those observed at $1.4 \mathrm{GHz}$. The arm-interarm contrast is lower at $151 \mathrm{MHz}$ than at $1400 \mathrm{MHz}$, indicating propagation of CRE from spiral arms into interarm regions. The correlations between the images of radio emission at $151 \mathrm{MHz}$ and $1400 \mathrm{MHz}$ and the FIR emission at $70 \mu \mathrm{m}$ reveal breaks on scales of 1.4 and $0.7 \mathrm{kpc}$, respectively. The total (equipartition) magnetic field strength decreases from about $28 \mu \mathrm{G}$ in the central region to about $10 \mu \mathrm{G}$ at $10 \mathrm{kpc}$ radius. No significant polarisation was detected from M 51, owing to severe Faraday depolarisation. Six extragalactic sources are detected in polarisation in the M51 field of $4.1^{\circ} \times 4.1^{\circ}$ size. Two sources show complex structures in Faraday space.

Conclusions. Our main results, the scale lengths of the inner and outer disks at $151 \mathrm{MHz}$ and $1.4 \mathrm{GHz}$, arm-interarm contrast, and the break scales of the radio-FIR correlations, can be explained consistently by CRE diffusion, leading to a longer propagation length of CRE of lower energy. The distribution of CRE sources drops sharply at about $10 \mathrm{kpc}$ radius, where the star formation rate also decreases sharply. We find evidence that thermal absorption is primarily caused by $\mathrm{H}$ II regions. The non-detection of polarisation from M 51 at $151 \mathrm{MHz}$ is consistent with the estimates of Faraday depolarisation. Future searches for polarised emission in this frequency range should concentrate on regions with low star formation rates.

Key words. polarization - cosmic rays - galaxies: ISM - galaxies: magnetic fields - radio continuum: galaxies

* The total intensity FITS file of the Stokes $I$ image of M 51 is only available at the CDS via anonymous ftp to cdsarc.u-strasbg.fr (130.79.128.5) or via http://cdsarc.u-strasbg.fr/viz-bin/qcat?J/A+A/568/A74 $\star \star$ Now at the School of Physics and Astronomy, University of Southampton, Highfield, SO17 1SJ, Southampton, UK.

$\star \star \star$ Now at the Leibniz-Institut für Astrophysik Potsdam, An der Sternwarte 16, 14482 Potsdam, Germany. 
Table 1. Physical parameters of M $51=$ NGC 5194.

\begin{tabular}{ll}
\hline \hline Morphology & SAbc \\
Position of the nucleus & $\alpha(2000)=13^{\mathrm{h}} 29^{\mathrm{m}} 52^{\mathrm{s} .709}$ \\
& $\delta(2000)=+47^{\circ} 11^{\prime} 42.59^{\prime \prime}$ \\
Position angle of major axis & $-10^{\circ}\left(0^{\circ}\right.$ is north $)$ \\
Inclination & $-20^{\circ}\left(0^{\circ} \text { is face on }\right)^{a}$ \\
Distance & $7.6 \mathrm{Mpc}^{b}$ \\
Optical radius $\left(R_{25}\right)$ & $3.9^{\prime}(8.6 \mathrm{kpc})^{c}$ \\
\hline
\end{tabular}

Notes. ${ }^{(a)}$ Tully (1974); ${ }^{(b)}$ Ciardullo et al. (2002); ${ }^{(c)}$ Bigiel et al. (2010).

\section{Introduction}

Messier 51, or NGC 5194, aptly named the "Whirlpool Galaxy", is one of the most widely studied galaxies because it is one of the most striking nearby examples of a classical grand-design spiral galaxy. Spiral galaxies exhibit a wide range of morphologies, from flocculent to grand-design, that can be caused by smallscale gravitational instabilities or caused by larger scale perturbations caused by global density waves, tidal interactions, or bars. In the case of M 51, many numerical simulations have been performed to study the consequences of the gravitational interaction between the main galaxy, NGC 5194, and its nearby companion NGC 5195 (Salo \& Laurikainen 2000; Theis \& Spinneker 2003). For example, Dobbs et al. (2010) used hydrodynamical models to simulate the tidally induced spiral structure. In these studies the authors were able to replicate several features seen in observations, such as the H I tidal tail (Rots et al. 1990) and kinks and bifurcations in the spiral arms. The physical parameters of M 51 used in this paper can be seen in Table 1 .

M51 is the first external galaxy where polarised radio emission was detected with the Westerbork Synthesis Radio Telescope, (WSRT; Mathewson et al. 1972). polarised emission is observed throughout most of the disk of M 51, as observed at $10.7 \mathrm{GHz}$ with the Effelsberg 100-m telescope by Neininger (1992), and the pattern of magnetic field lines follows that of the optical spiral arms. Horellou et al. (1992) found from VLA observations at frequencies of 1.47 and $1.67 \mathrm{GHz}$ that the distribution of polarised emission is strongly affected by Faraday depolarisation and that the polarised emission is actually emerging from an upper layer of the disk, because the galaxy is not transparent to polarised emission at these frequencies. Heald et al. (2009) again observed polarisation from M 51 at $1.30-1.76 \mathrm{GHz}$ throughout the whole disk; however, it was seen that there were large variations with a $5 \%$ polarisation fraction detected in the optical disk and 25-30\% beyond the outer arms. Heald et al. (2009) also performed RM Synthesis on these data and detected a main Faraday component at $+13 \mathrm{rad} \mathrm{m}^{-2}$, with the Faraday rotation dominated by Milky Way foreground. Braun et al. (2010) made additional use of this technique and studied two weaker secondary components near -180 and $+200 \mathrm{rad} \mathrm{m}^{-2}$, coming from M 51 itself.

High-resolution VLA observations at $4.86 \mathrm{GHz}$, combined with Effelsberg data at similar frequencies (Fletcher et al. 2011), have shown that the locations of the inner radio spiral arms coincide with $\mathrm{CO}$ emission, while the polarised emission peaks at the inner edge of the material arms (Patrikeev et al. 2006). The polarised emission mostly emerges from anisotropic turbulent fields generated by compressing and shearing gas flows. Schinnerer et al. (2013) found evidence in M 51 for a physical link between the molecular gas as observed in the CO line and the total magnetic field seen in the total radio continuum emission, both at $2^{\prime \prime}$ resolution.
From the analysis of VLA plus Effelsberg polarisation observations at 4.86 and $8.46 \mathrm{GHz}$, Fletcher et al. (2011) were able to identify two underlying patterns of regular magnetic fields. A regular field with a combination of $m=0+2$ azimuthal modes was found in the disk, while in the halo a regular field with a bisymmetric $m=1$ azimuthal mode predominates. The origin of this regular field is probably due to two large-scale dynamos (Beck et al. 1996) operating in the disk and the halo.

Considering how extensively M 51 has being studied both observationally and theoretically, very few observations have being performed on M 51 at frequencies below $500 \mathrm{MHz}$, primary due to the difficulties of observing at low radio frequencies. Low-frequency radio synchrotron emission is very important as it is produced by aged and low-energy electrons that are less affected by energy losses and therefore can propagate further away from their site of origin (assuming that the diffusion coefficient does not vary with particle energy). Depending on the magnetic field strength in the outer disk, one would expect to see a large radio synchrotron disk around M 51. The extent and profile of the radio disk at low frequencies contains information about the propagation of cosmic ray electrons (CRE) and the strength of the magnetic field beyond the disk of strong star formation. This will be investigated in this paper. Segalovitz (1977) observed M 51 with the WSRT at $610 \mathrm{MHz}$ as well as $1.4 \mathrm{GHz}$, but with a resolution of approximately $1^{\prime}$ for the $610 \mathrm{MHz}$ observation, only few details of the inner galaxy could be observed. An extended component could be seen but this extended no further than the $1.4 \mathrm{GHz}$ observation. Using both observations Segalovitz (1977) computed a spectral index image which was compared with analytical solutions of the cosmic ray diffusion equation. Two sets of parameters produced models which could fit the observations, namely: (a) a constant magnetic field and diffusion coefficient with leakage of electrons out of the galactic magnetic field perpendicular to the disk; or (b) no leakage but a magnetic field that is proportional to $1 / r^{1 / 2}$ and a diffusion coefficient that is proportional to $1 / r$, where $r$ is the galactocentric distance. However, the step-like source function used in the analytical solutions is now outdated and unrealistic.

M 51 was also observed at low frequencies as part of a survey of 133 spiral galaxies performed by (Israel \& Mahoney 1990, hereafter I \& M) using the Clarke Lake Telescope at $57.5 \mathrm{MHz}$ with a total flux density of $11 \pm 1.5 \mathrm{Jy}$. However these results may suffer from uncertainties. Overall, the measured total flux densities in nearly all galaxies of this survey were lower than expected through extrapolating from higher frequencies. In addition, it was found that more highly inclined galaxies had lower fluxes than moderate-inclined galaxies. I\&M explained this flattening of the radio spectrum through free-free absorption, especially because of its dependence on inclination. Such an effect may indicate the existence of a clumpy medium of well-mixed, non-thermally emitting and thermally absorbing gas with a small filling factor and an electron temperature of 500 to $1000 \mathrm{~K}$. Hummel (1991) reanalysed the same data and confirmed the spectral index flattening, but found that the magnitude of the flattening does not depend on the inclination of the galaxy. This is one of the main issues that needs to be addressed with low-frequency observations, especially with the upcoming Multifrequency Snapshot Sky Survey (MSSS; Heald et al. in prep.).

Little is known about the conditions in the interstellar medium (ISM) of the outer disk ( $\left.r>R_{25}\right)$. Thornley et al. (2006), using IR, UV, $\mathrm{H} \alpha$ and $\mathrm{H}$ I measurements, found that star formation in the outer disk of M 51 is about an order of magnitude less efficient than at smaller radii. Braine \& Herpin (2004) observed 
carbon monoxide (CO) in the outer disk of NGC 4414 in regions of high H I column density regions. In these regions, Bigiel et al. (2010) found that despite widespread star formation, the outer disk star formation is extremely inefficient, that is, the gas depletion time is very low and increases with radius. A low rate of star formation means that the level of turbulence is probably too low to maintain a strong magnetic field (Beck et al. 1996). On the other hand, turbulence could also be induced by the magnetorotational instability (Sellwood \& Balbus 1999) in the outer regions of the galaxy, thereby increasing the turbulent energy density, magnetic field strength, and hence the scale length of radio synchrotron emission (Beck 2007).

We shall outline the results from the first LOFAR (van Haarlem et al. 2013) observations of a nearby galaxy, namely M 51. In Sect. 2 we will describe the observational setup along with the data reduction process. In Sect. 3 we will present the LOFAR HBA $151 \mathrm{MHz}$ image of M51 and investigate the radial profile of LOFAR emission while comparing to higher frequencies. In Sects. 4 and 5 we will present and discuss the spectral index and total magnetic field strength images derived from the LOFAR $151 \mathrm{MHz}$ and VLA $1.4 \mathrm{GHz}$ image from Fletcher et al. (2011). In Sect. 6, the cosmic ray propagation in M 51 will be discussed, and the wavelet cross correlation between radio and far-infrared (FIR) emission will be shown in Sect. 7. In Sect. 8 we shall also present results from performing rotation measure synthesis on this region and the analysis of detected background sources in the field. Finally, conclusions and prospects for future work will be presented in Sects. 9 and 10.

\section{Observations and preprocessing data reduction}

The M51 field was observed for $8 \mathrm{~h}$ with a configuration of 61 LOFAR High Band Antenna (HBA) fields - 48 core station fields and 13 remote station fields. No international stations were used in this observation because sub-arcsec resolution was not needed and adding them would have required the removal of some core stations. The observation was done in dual beam mode, with one beam targeting M51 $(\alpha=$ $\left.13^{\mathrm{h}} 29^{\mathrm{m}} 52^{\mathrm{s}} 7, \delta=+47^{\circ} 11^{\prime} 43^{\prime \prime}\right)$ and one beam targeting $3 \mathrm{C} 295$ $\left(\alpha=14^{\mathrm{h}} 11^{\mathrm{m}} 20^{\mathrm{s}} 5, \delta=+52^{\circ} 12^{\prime} 10^{\prime \prime}\right)$ simultaneously. This could be done because 3C295 is less than 10 degrees from M51 and thus within the HBA analog tile beam which was centred on M51 itself. The second beam was used for flux and initial phase calibration. The so-called 8-bit mode ${ }^{1}$ of LOFAR was used, giving an instantaneous bandwidth of about $95 \mathrm{MHz}$. This total bandwidth is split into 488 subbands, each with a bandwidth of $0.195 \mathrm{MHz}$. In this observation the subbands were divided evenly between the calibrator (244 subbands) and the target (244 subbands). As these 244 subbands cannot fully cover the HBA band, the bandwidth in each beam was split into 8 blocks of approximately 30 subbands (about $6 \mathrm{MHz}$ bandwidth) spread evenly over the HBA band. The observation was done between $115 \mathrm{MHz}$ and $175 \mathrm{MHz}$ to avoid the band edge of the analog filters at the low frequencies and the strong $\mathrm{RFI}^{2}$ from $\mathrm{DAB}^{3}$ above $175 \mathrm{MHz}$ (Offringa et al. 2013). Details of the observations are given in Table 2.

The majority of the data reduction was performed with the LOFAR pipeline. A full description of this pipeline is out of the

\footnotetext{
1 The data being sent by the LOFAR stations is encoded with 8-bit integers.

2 Radio frequency interference.

3 Digital audio broadcast.
}

Table 2. Parameters of the M 51 LOFAR observations.

\begin{tabular}{ll}
\hline \hline Start date (UTC) & 22-Apr.-2013/20:08:07.0 \\
End date (UTC) & 23-Apr.-2013/04:04:04.7 \\
\hline Frequency range & $115.9-175.8 \mathrm{MHz}$ \\
Total bandwidth on target & $47.7 \mathrm{MHz}$ \\
Total bandwidth on calibrator & $47.7 \mathrm{MHz}$ \\
\hline
\end{tabular}

scope of this paper. Interested readers can refer to Heald et al. (2010) and van Haarlem et al. (2013).

RFI excision was done with the AOFlagger pipeline (Offringa et al. 2012), which was used on the raw data and all linear correlations to give the best results for RFI detection and flagging. The visibility data were checked to see if any contamination from the so called A-Team (Cassiopeia A, Cygnus A, Virgo A, and Taurus A) entered though the sidelobes. Only minimal contamination was seen, so "demixing" (van der Tol et al. 2007) was not applied, but affected visibilities were flagged. The data were then compressed to 8 channels per subband in frequency and $14 \mathrm{~s}$ sampling time. These flagging and compression operations were performed using the New Default Pre-Processing Pipeline (NDPPP), which is part of the LOFAR software.

\subsection{Initial calibration}

The calibrator subbands of 3C 295 were calibrated using a sky model from Scaife \& Heald (2012) using the Black Board Selfcal (BBS) software (Pandey et al. 2009). Note that the flux scale is that of (Roger et al. 1973, hereafter RBC). This scale is used to avoid the suggested issues (Rees 1990) with the secular decrease in the flux density of Cassiopeia A at low frequencies existing in the Baars et al. (1977) scale.

The calibration solutions found from calibrating the 3C 295 data were directly transferred to the corresponding target data at the same frequency. This included the phase solutions as it made the next phase only calibration easier.

The target subbands for each block of approximately 30 subbands were combined in frequency for a better signal to noise ratio. These blocks were then phase calibrated using the preliminary LOFAR global sky model. This global sky model consists of positions of sources from the NRAO VLA Sky Survey (NVSS) catalogue (Condon et al. 1998) with fluxes obtained from a power-law spectral fitting with data from the NVSS, the Westerbork Northern Sky Survey (WENSS; Rengelink et al. 1997) and the VLA Low-Frequency Sky Survey redux (VLSSr; Cohen et al. 2007) (Lane et al. 2014) surveys.

After this initial calibration, each block of subbands was checked manually for any RFI or bad solutions resulting from the calibration, which were in turn flagged.

\subsection{Self calibration}

Once the initial direction-independent calibration had been completed, a new sky model was created from images produced by two programs. First, an image of the full field-of-view was created using AWimager (Tasse et al. 2013) which is part of the LOFAR software. This program utilises the A-projection algorithm (Bhatnagar et al. 2008), when imaging wide fields to correct for direction-dependent effects resulting from changing primary beams and ionospheric effects. It can therefore yield accurate fluxes for sources far away from the phase centre. Next, a 


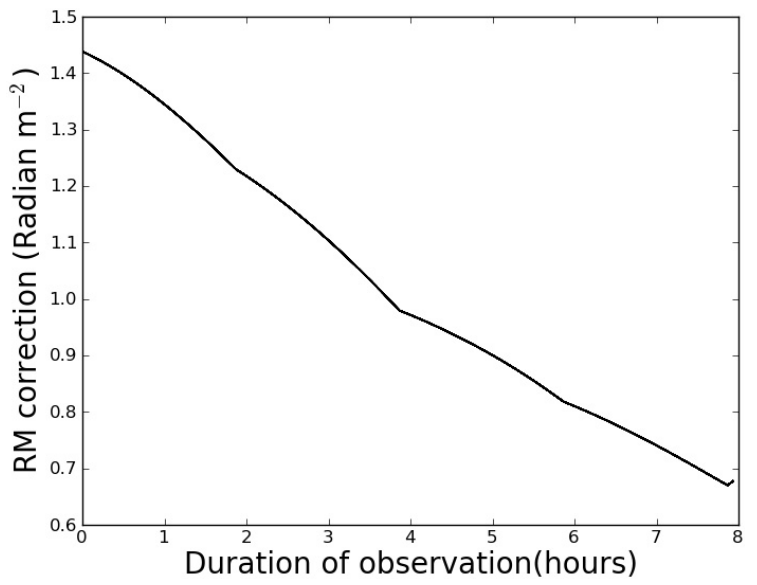

Fig. 1. Calculated ionospheric RM correction in the direction of M51 for the duration of the observation.

second image was created using $\mathrm{CASA}^{4}$ and utilising its multiscale clean (Cornwell 2008) and w-projection (Cornwell et al. 2005) tasks solely for creating a detailed model for M 51 itself. Multiscale clean is needed to accurately image the extended structures in M 51 and was not available in the AWimager at the time of processing.

As M 51 is very small compared to the size of the primary beam and is located at the phase centre of our observation, errors due to the missing primary beam correction are small. Both images were made with an uv coverage of $12 \mathrm{k} \lambda$, which corresponds to 20-25" resolution with uniform weighting. The clean components for M51 from the CASA image and the rest of the field from the AWimager were combined into the new sky model. That was then used for direction-independent self calibration of the phases. This whole process was performed on each of the 8 blocks of 30 subbands. No sources were subtracted from the visibilities during the calibration.

At low frequencies, correction for ionospheric Faraday rotation is needed in order to avoid depolarisation caused by the changing ionosphere. Values of the RM correction were calculated using the method by Sotomayor-Beltran et al. (2013). Measurements of the vertical total electron content (VTEC) were obtained from the centre for Orbit Determination in Europe (CODE) which have a time resolution of $2 \mathrm{~h}$. These were combined with the Earth's magnetic field as calculated using the eleventh generation of the International Geomagnetic Reference Field (IGRF11; Finlay et al. 2010). The calculated RM correction in the direction of M 51 was applied to the data. The values for the entire observation can be seen in Fig. 1.

\section{M 51 total power image}

Images were cleaned using the same imaging parameters and uv-distance (namely $16 \mathrm{k} \lambda$ ) for each of the eight subband blocks with CASA in order to utilise multi-scale clean with wprojection. A mask was used in this cleaning which was created by hand. Several point sources were compared to the point spread function (PSF) to investigate if the point sources were broadened due to phase errors caused by the ionosphere, but no significant broadening could be seen. Also, the astrometry of these point sources was checked with catalogue values, but no major deviations could be seen.

\footnotetext{
4 http://casa.nrao.edu/
}

All eight frequency block images were averaged in the image plane using an inverse variance weighting scheme. The central frequency is $151 \mathrm{MHz}$. The final image of M 51 at 20" resolution with robust weighting of -0.5 is shown in Figs. 2 and 3 which shows the extended disk well.

The rms noise in quiet regions close to the edges of the field of view $(\mathrm{FoV})$ for both uniform and robust weighting schemes is approximately $150 \mu \mathrm{Jy} /$ beam. Closer to M 51 the noise in the image with uniform weighting is between 200 and $300 \mu \mathrm{Jy} /$ beam while the noise in the robust image (Fig. 3) is between 300 and $400 \mu \mathrm{Jy} /$ beam. The theoretical thermal noise value is expected to be about $30 \mu \mathrm{Jy} /$ beam or taking into account the weighting scheme, $60 \mu \mathrm{Jy} /$ beam. This means we achieve approximately $2.5-5$ times the thermal noise. This is the deepest image obtained so far for any galaxy in the low-frequency regime $(<300 \mathrm{MHz})$. Phase errors are seen around the brighter sources in the field but these are localised. No phase errors are obvious near M 51. Directional-dependent calibration would need to be performed in many directions to get rid of these phase errors completely. This will be done in a later work when studying M 51 at much higher resolution.

The grand design spiral arms of M 51 still can be clearly seen at $151 \mathrm{MHz}$. The arm and interarm contrast is highly visible in M 51 at these low frequencies and comparable at first glance to higher frequencies (Fletcher et al. 2011). This is in stark contrast to the galaxies observed at $333 \mathrm{MHz}$ with the GMRT where the spiral arms and inter arm regions were often indiscernible from each other (Basu et al. 2012a). According to Fletcher et al. (2011), this is primarily caused by the low-energy CREs diffusing from star forming regions in the spiral arms without losing much energy compared to higher energy electrons seen at higher frequencies. This may enable the lower energy electrons to diffuse further and thus fill up the interarm regions.

CRE diffusion from the spiral arms into the interarm region can be seen in the $151 \mathrm{MHz}$ image when compared to images at higher frequencies. Especially in the northern region, the interarm region is becoming squeezed as the CRE diffuse into the interarm region. However, it is not as severe compared to the galaxies observed by Basu et al. (2012a).

The region between the companion and the northern arm of NGC 5194 where little IR emission exists (Dumas et al. 2011) is very distinct at $151 \mathrm{MHz}$. It is much brighter compared to the 4.86 and $8.46 \mathrm{GHz}$ images by Dumas et al. (2011) and Fletcher et al. (2011), indicative of synchrotron emission that is enhanced by the ongoing interaction. This is seen in Sect. 4 where the spectral index is larger than -0.8 .

Supernova SN 2011dh, discovered on 01 June 2011 by the Palomar Transient Factory project (PTF; Arcavi et al. 2011), (Silverman et al. 2011), was not detected in our observations. This gives an upper limit of the SN flux density at $151 \mathrm{MHz}$ at day 691 of $11.3 \pm 0.7 \mathrm{mJy} / \mathrm{beam}$.

Figure 3 shows the full extent of the disk of M 51 as far out as $16 \mathrm{kpc}$ away from the galactic centre. This is the largest extent of M 51 detected so far in radio continuum. The emission is not increasing uniformly with radius. An extension of the disk to the northeast is possibly caused by an outflow generated by the interaction with the companion.

\section{Spectral properties of M 51}

\subsection{Integrated radio continuum spectrum}

To calculate the flux density of M51 we integrated the total emission in concentric rings whilst taking into account the 


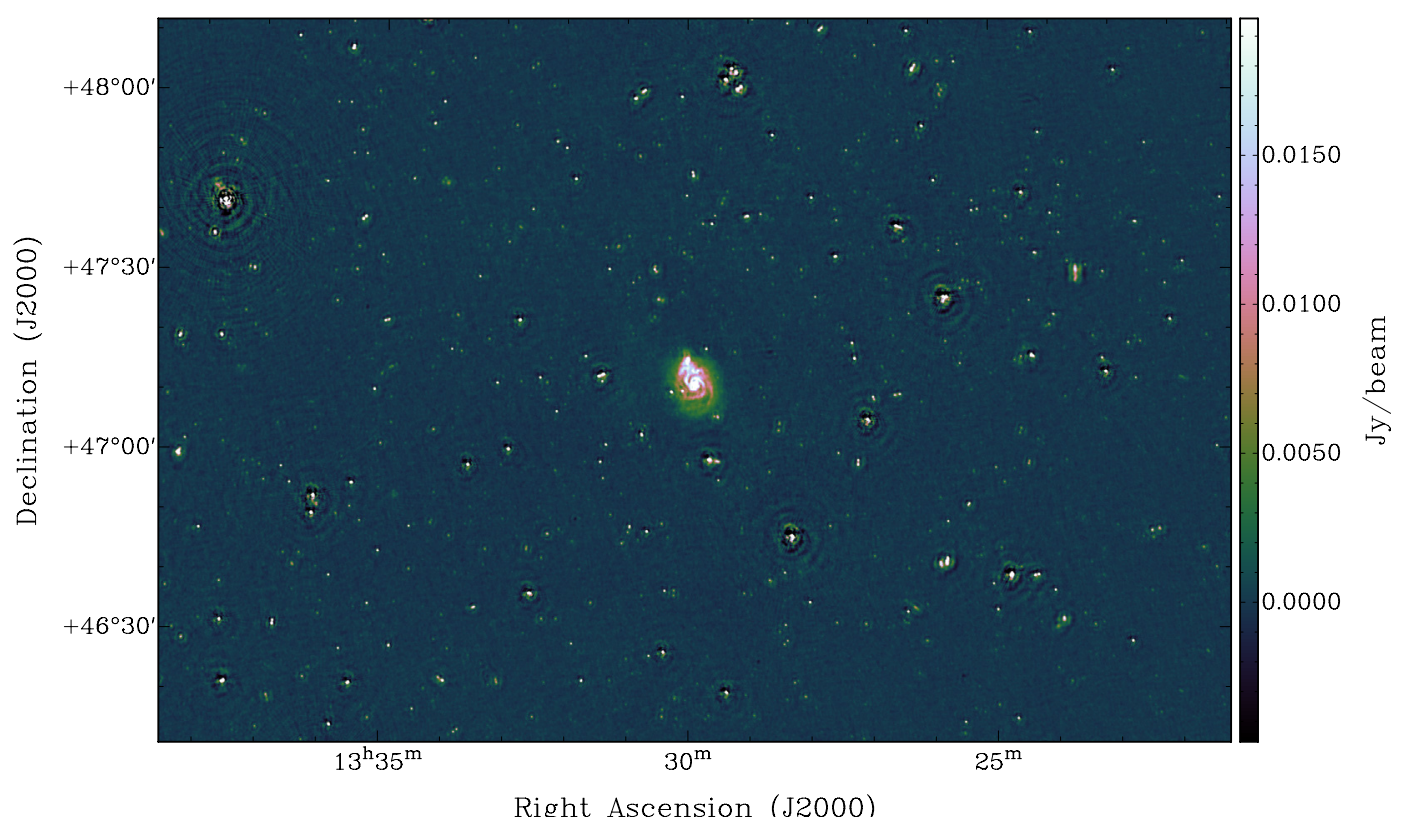

Fig. 2. Region around M 51 at a central frequency of $151 \mathrm{MHz}$ with a bandwidth of $47.7 \mathrm{MHz}$. The resolution is $20^{\prime \prime}$ and a robust weighting of -0.5 was used. The colour scale is in Jy/beam using a cubehelix colour scheme (Green 2011).

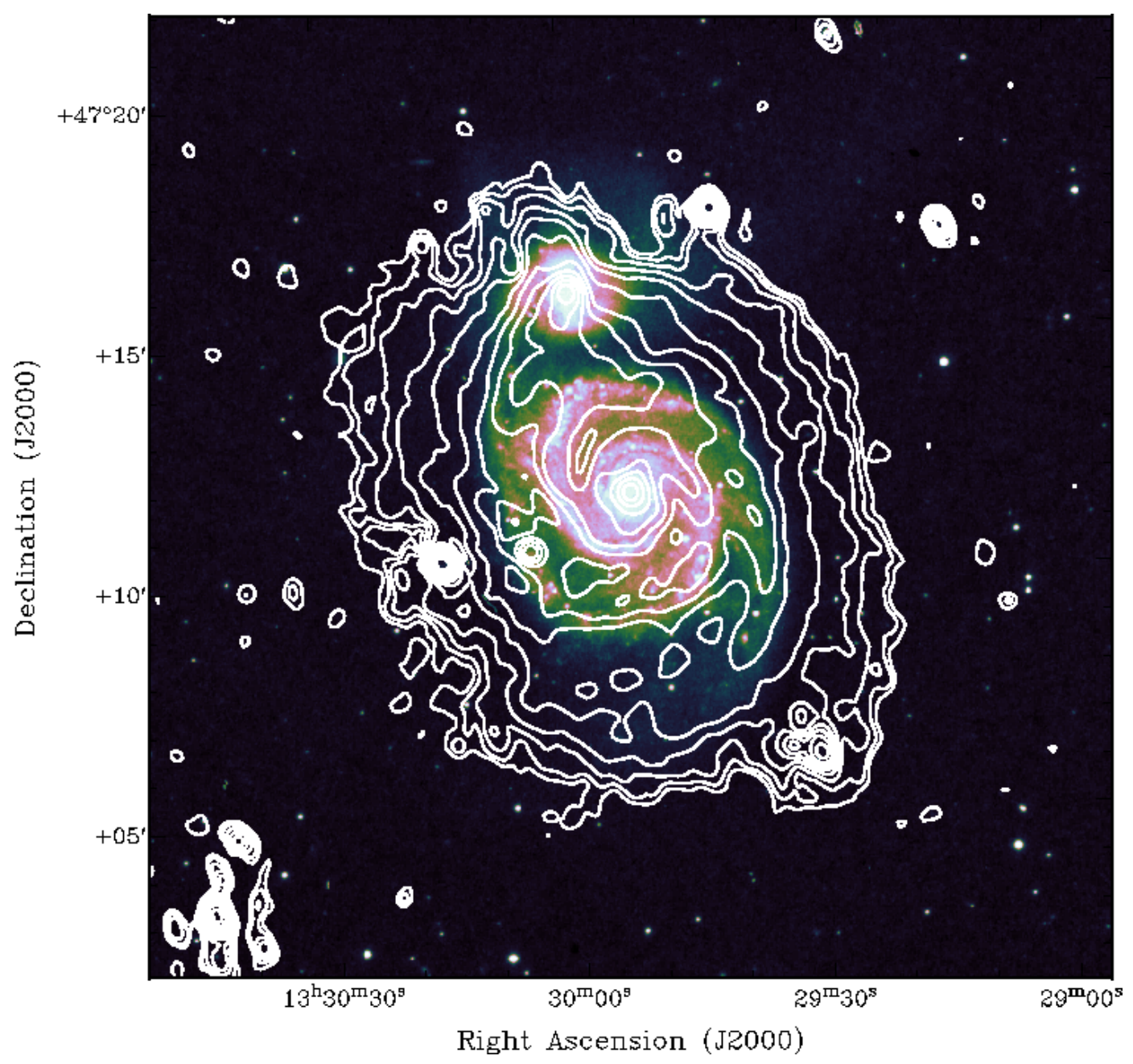

Fig. 3. M 51 at a central frequency of $151 \mathrm{MHz}$ with a bandwidth of $47.7 \mathrm{MHz}$ overlayed onto an optical DSS image using a cubehelix colour scheme (Green 2011). The resolution is $20^{\prime \prime}$ and a robust weighting of -0.5 was used. Here the extended disk is seen clearly. The contours start at $1 \mathrm{mJy} /$ beam and increase by a factor 1.5 . 
Table 3. Integrated flux densities for M 51 from literature, rescaled to the RBC scale, which are used for the fit in Fig. 4.

\begin{tabular}{lcc}
\hline \hline$v(\mathrm{GHz})$ & Flux density $(\mathrm{Jy})$ & Ref. \\
\hline 22.8 & $0.147 \pm 0.016$ & 1 \\
14.7 & $0.197 \pm 0.021$ & 1 \\
10.7 & $0.241 \pm 0.014$ & 2 \\
8.46 & $0.308 \pm 0.103$ & 3 \\
4.86 & $0.604 \pm 0.201$ & 4 \\
2.604 & $0.771 \pm 0.049$ & 1 \\
1.49 & $1.36 \pm 0.09$ & 4 \\
0.61 & $2.63 \pm 0.06$ & 5 \\
0.408 & $3.5 \pm 0.1$ & 6 \\
0.15 & $6.9 \pm 0.69$ & 7 \\
0.15 & $6.48 \pm 0.65$ & 8 \\
0.15 & $8.1 \pm 0.6$ & This work \\
\hline
\end{tabular}

References. (1) Klein et al. (1984); (2) Klein \& Emerson (1981); (3) Dumas et al. (2011); (4) Fletcher et al. (2011); (5) Segalovitz (1977); (6) Gioia \& Gregorini (1980); (7) Hales et al. (1988); (8) Waldram et al. (1996).

major axis and inclination of the galaxy's plane projected on the sky. We obtained an integrated flux density measurement of $S_{151}=8.1 \pm 0.6 \mathrm{Jy}$ out to a radius of $16 \mathrm{kpc}$. The star forming region $(r<10 \mathrm{kpc})$ contains $6.98 \mathrm{Jy}$ and the extended disk $(r>10 \mathrm{kpc})$ contains approximately $1.1 \mathrm{Jy}$ of flux density.

We used other measurements from higher frequencies, given in Table 3, to compute the spectral index of M51. As we have used the RBC flux scale for scaling our LOFAR data, measurements at other frequencies must be rescaled. Fortunately, at frequencies $\gtrsim 300 \mathrm{MHz}$, the RBC scale is in agreement with KPW scale (Kellermann et al. 1969) and therefore we can use the conversion factors from the Baars scale (Table 7 in Baars et al. 1977).

Comparing to previous flux measurements, the LOFAR flux density agrees very well with other lower frequency data as well as to the higher frequency data. The 4.86 and $8.46 \mathrm{GHz}$ values by Fletcher et al. (2011) have significant uncertainties due to addition of single dish data to interferometry data. The integrated flux of M51 from the 6C (Hales et al. 1988) $(6.90 \pm 0.69 \mathrm{Jy})$ and 7C (Waldram et al. 1996) (6.48 $\pm 0.65 \mathrm{Jy})$ surveys are very comparable the flux of the star forming region of M 51 (6.98 Jy).

We fit a single power law with a spectral index of $\alpha=-0.79 \pm 0.02$ (Fig. 4). There is no indication of flattening of the integrated spectrum for M 51 at low frequencies down to $151 \mathrm{MHz}$ due to thermal absorption or ionisation losses. The flattening towards $57.5 \mathrm{MHz}$ is uncertain, especially when compared to the flux measurement of $26.3 \mathrm{MHz}$. The spectral index between 26.3 MHz and our LOFAR flux is approximately $\alpha=$ -0.76 , showing no significant flattening of the spectrum down to $26.3 \mathrm{MHz}$. This shows the importance of upcoming LOFAR LBA data. Towards high frequencies no flattening by thermal emission (with a spectral index of -0.1) is observed. The average thermal fraction at $4.86 \mathrm{GHz}$ is about $25 \%$ from the observed spectral index and assuming a constant synchrotron spectral index across the galaxy disk (Fletcher et al. 2011), which is an overestimate if the synchrotron spectral index is flatter in the spiral arms (Tabatabaei et al. 2007b), and about 12\% when integrating the luminosity function of the H II regions (van der Hulst et al. 1988). Assuming $16 \%$ at $4.86 \mathrm{GHz}$, this should increase to $36 \%$ at $22.8 \mathrm{GHz}$ and flatten the spectral index between 4.86 and $22.8 \mathrm{GHz}$ to -0.63 , which is clearly not observed. The flattening is possibly cancelled by a steepening

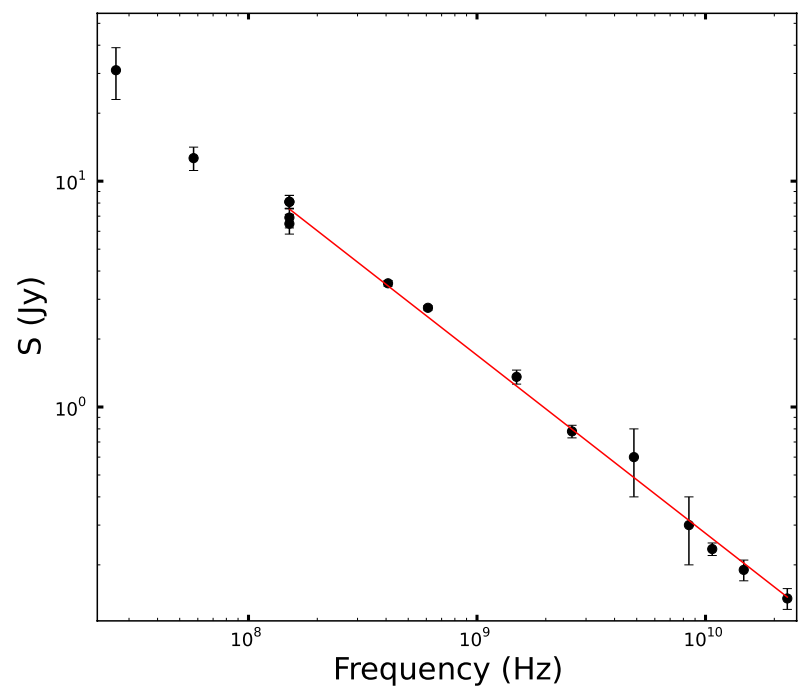

Fig. 4. Integrated flux density of M 51 with a power law fit of slope $\alpha=-0.79 \pm 0.02$. The integrated flux values of $11 \pm 1.5 \mathrm{Jy}$ from Israel \& Mahoney (1990) at $57.5 \mathrm{MHz}$ and $31 \pm 8 \mathrm{Jy}$ at $26.3 \mathrm{MHz}$ (Viner \& Erickson 1975) are also plotted.

of the nonthermal synchrotron spectrum by synchrotron and inverse Compton losses (see discussion in Sect. 9). The steepening of the synchrotron spectrum should become significant beyond $2 \mathrm{GHz}$, where the average thermal fraction exceeds $10 \%$. Another possibility is a lack of detected flux density at the highest frequencies listed in Table 3 where the signal-to-noise ratios are lowest. This makes future observations of galaxies at frequencies above $20 \mathrm{GHz}$ vital.

\subsection{Spectral index map of $M 51$}

A spectral index image was created from the VLA image of M 51 at $1.4 \mathrm{GHz}$ and the $151 \mathrm{MHz}$ LOFAR image. Both images were made with the same uv distance (namely 0.1 to $16 \mathrm{k} \lambda$ ). The $1.4 \mathrm{GHz}$ image was convolved to $20^{\prime \prime}$ resolution and placed onto the same grid as the $151 \mathrm{MHz}$ LOFAR image. Nearby point sources were either subtracted by fitting a Gaussian component and then subtracting or (for sources within M 51) manually blanked out. Only pixels that are above $5 \sigma$ level in both images were used. The spectral index was computed pixel by pixel and is shown in Fig. 5 along with the uncertainty image.

A contrast between spiral arm and interarm regions is obvious in the spectral index image, indicating energy losses of the CREs as they propagate from the star forming regions into the interarm regions. According to the H II overlay (Fig. 6), regions of strong star formation in the spiral arms show flat spectral indices. Regions of high star formation are bulging into the interarm region, specifically in the inner southeastern region where several $\mathrm{H}$ II regions exist in the interarm regions (Lee et al. 2011).

\subsection{Spectral radial profile of $M 51$}

Figure 7 shows the radial spectral index profile, created from the mean flux densities at $1.4 \mathrm{GHz}$ and $151 \mathrm{MHz}$ in concentric rings in the galaxy's plane and then computing the spectral index. The depressions around $r \sim 2 \mathrm{kpc}$ and a larger dip at $r \sim 3.5 \mathrm{kpc}$ are associated with the interarm regions of M 51. The spectral index rises again as the spiral arm region becomes more dominant. In the range $4 \mathrm{kpc}<r \leq 10 \mathrm{kpc}$ the spectral index gently decreases with a slight upturn at around $r \sim 10 \mathrm{kpc}$, signifying the location 

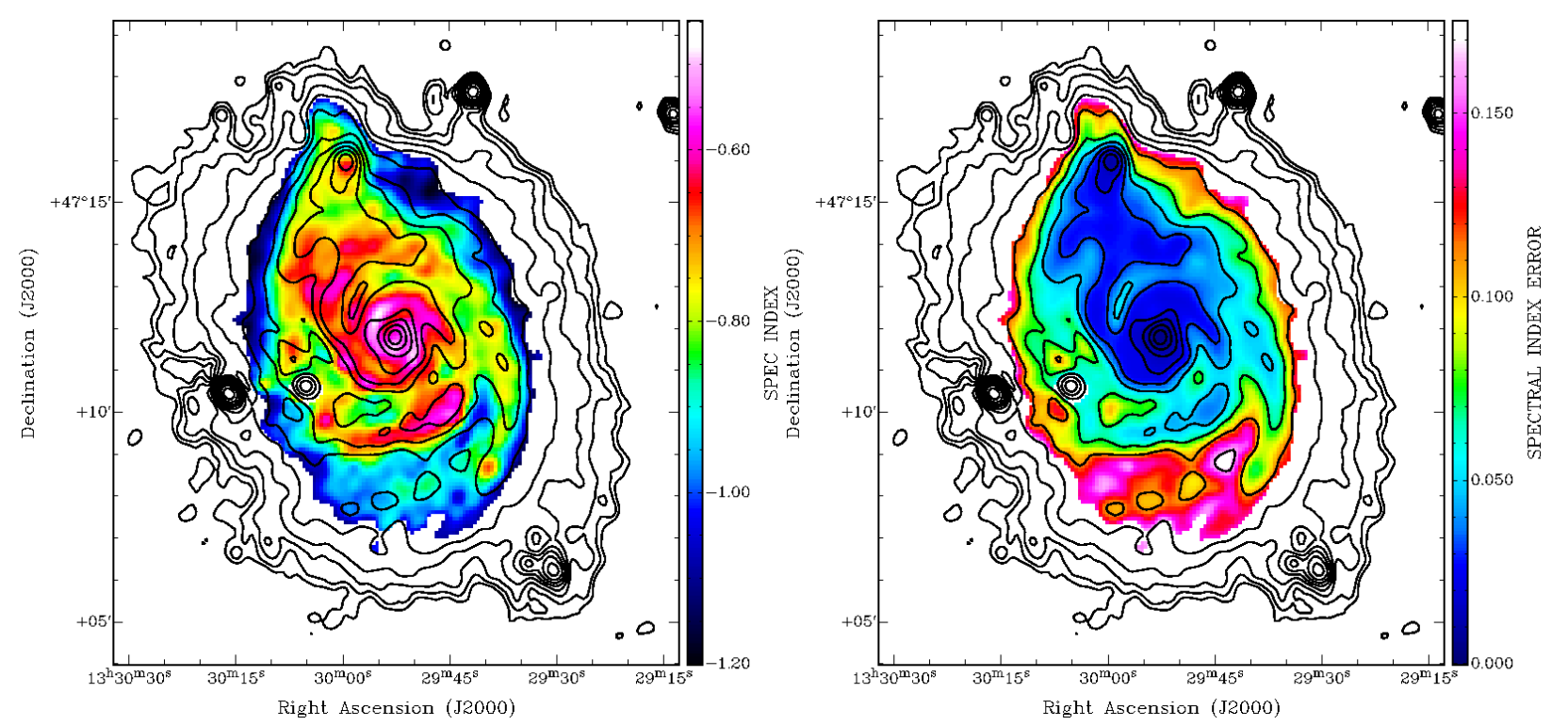

Fig. 5. Spectral index image (left) and spectral index error image (right) made from the $1.4 \mathrm{GHz}$ and $151 \mathrm{MHz}$ images at $20^{\prime \prime}$ resolution. The $151 \mathrm{MHz}$ image is overlayed with the same contour levels as Fig. 3.

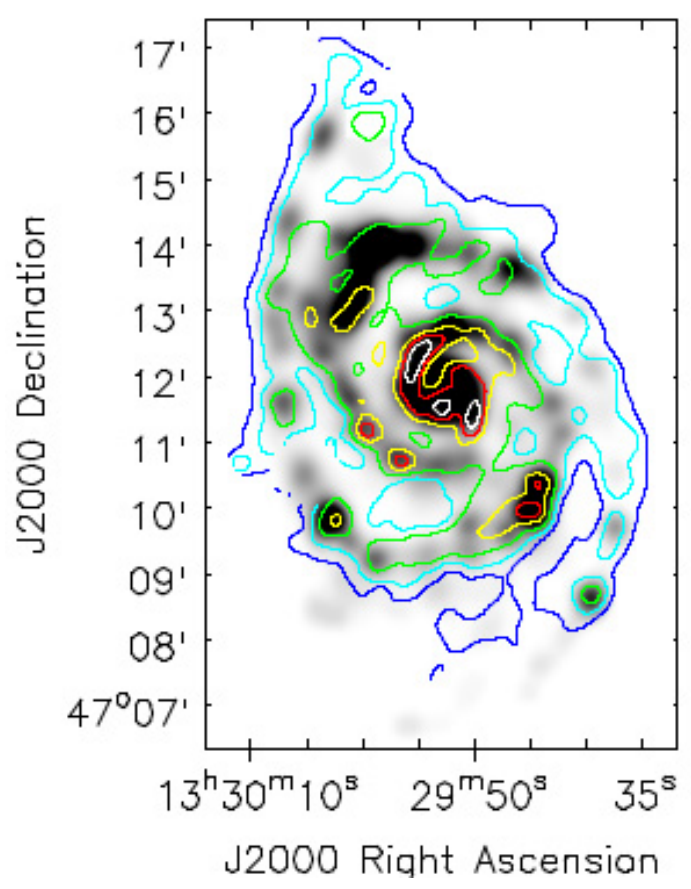

Fig. 6. H II from Scoville et al. (2001) smoothed to $20^{\prime \prime}$ resolution, with the map of spectral indices between $1.4 \mathrm{GHz}$ and $151 \mathrm{MHz}$ overlayed with contours. The white, red, yellow, green, cyan and blues contours show $-0.5,-0.55,-0.6,-0.7,-0.8,-0.9$, respectively.

of the companion galaxy NGC 5195. At $r>10 \mathrm{kpc}$ the spectral index drops rapidly, indicating that CREs are significantly older in the outer disk compared to the inner star forming regions of the galaxy.

The extended disk shows $\alpha \leq-0.9$, indicating energy losses through synchrotron cooling and inverse Compton losses. Figure 7 reveals a sharp decrease in $\alpha$ beyond $10 \mathrm{kpc}$, where the star formation rate is one order of magnitude smaller than in the central disk (Thornley et al. 2006), resulting in hardly any fresh injection of CREs.

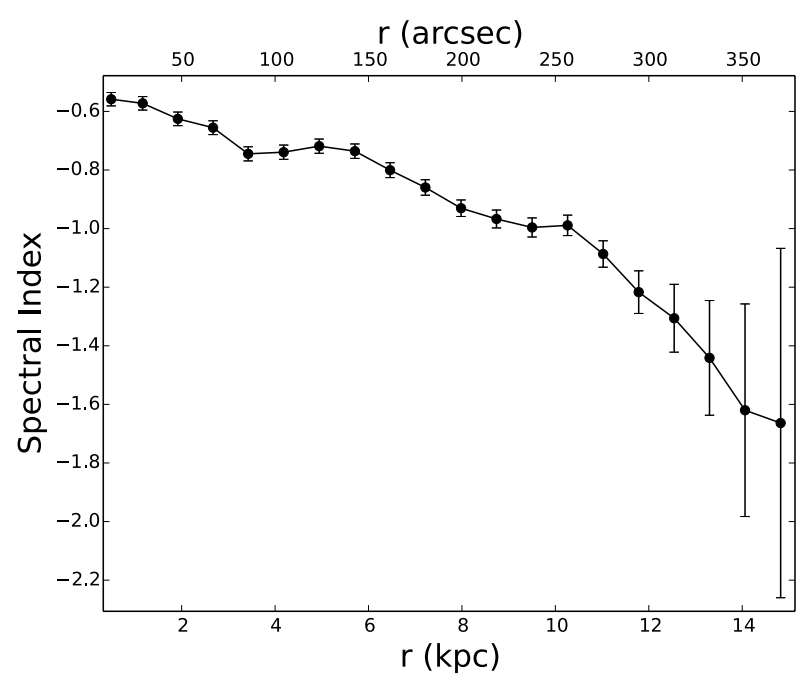

Fig.7. Radial profile of the spectral index between $1.4 \mathrm{GHz}$ and $151 \mathrm{MHz}$.

The outer disk of M51 towards the companion galaxy NGC 5195 behaves differently. The region between the companion and the northern arm of M 51 has a spectral index of $\sim-0.8$. The northern spiral arm continues far beyond the optical arm to the left of the companion. The arc shaped structure located just below NGC 5195 seen in radio continuum (Dumas et al. 2011) and in H II (Greenawalt et al. 1998) has a spectral index of $\sim-0.74$. These results indicate that CREs are injected locally in the region around the companion galaxy, for example by shock fronts generated by the interaction.

In contrast, the outer disk in the south has a spectral index of about -1.0 , causing a secondary peak in the spectral index histogram shown in Fig. 8, which can be explained by CRE diffusion from the spiral arms. The median value $(-0.82)$ of the spectral index found in Fig. 8 agrees quite well with the integrated spectrum of M 51 with $\alpha=-0.79 \pm 0.02$. 


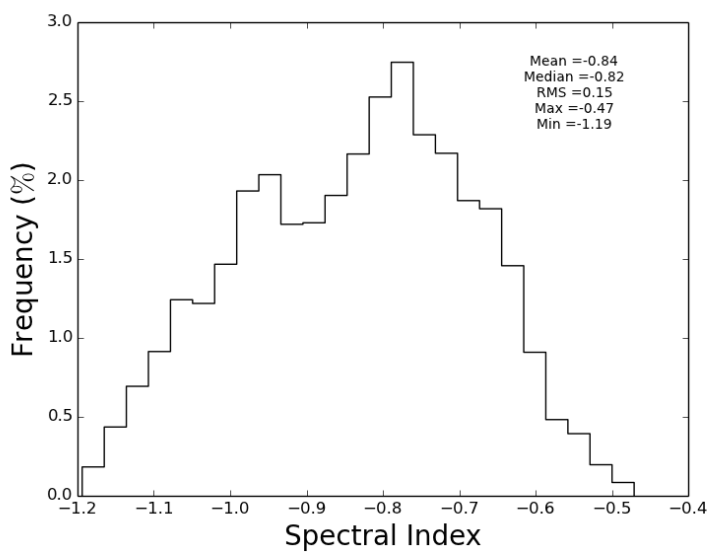

Fig. 8. Spectral index distribution grouped into 25 bins.

\subsection{Evidence of free-free absorption in M51}

Primary CREs are believed to be injected into galaxies via supernova remnants (SNR) with a power-law spectra $Q(E) \propto E^{p}$ where the spectral index $p$ of the energy spectrum is related to the spectral index $\alpha$ of the radio synchrotron emission via $\alpha=(p-1) / 2$ and $Q(E)$ is the CR source term. Models of diffusive shock acceleration in strong shocks predict $p \sim-2$ (Bell 1978a), consistent with observational results based on Galactic SNR from Kothes et al. (2006) and Green (2009) suggesting a mean nonthermal spectral index of $\alpha \sim-0.5$, which is indicative of a mature adiabatically expanding SNRs (Kothes et al. 2006). The energy spectrum of CRE, in the ISM is steepened to $p \sim-2.6$ due to energy dependent diffusion (Jokipii \& Higdon 1979), yielding a nonthermal radio spectral index of $\alpha \sim-0.8$.

We observe a spectral index of $-0.47 \geq \alpha \geq-0.52$ in the central region and inner spiral arms of M 51 (see Figs. 8 and 6), which is flatter than expected from CRE acceleration models. Using the following equation we can calculate the thermal fraction at $151 \mathrm{MHz}$ :

$f_{\mathrm{th}}=\frac{q^{\alpha}-q^{\alpha_{\mathrm{syn}}}}{q^{\alpha_{\mathrm{th}}}-q^{\alpha_{\mathrm{syn}}}}$,

where $q$ is the ratio of the two frequencies $(1400 \mathrm{MHz}$ and $151 \mathrm{MHz}), \alpha$ is the mean spectral index observed between the two frequencies, $\alpha_{\text {syn }}$ is the assumed synchrotron spectral index and $\alpha_{\text {th }}$ is the assumed thermal spectral index. If the flattening is caused entirely by free-free emission, $f_{\text {th }}=20-30 \%$ at $151 \mathrm{MHz}$ would be needed, assuming spectral indices of -0.8 for the synchrotron and -0.1 for the thermal emission. This is unlikely at such a low-frequency, because a similar thermal fraction has been found at $4.86 \mathrm{GHz}$ (Fletcher et al. 2011), while the thermal fraction must strongly decrease towards lower frequencies.

Scoville et al. (2001) found $1373 \mathrm{H}$ II emission regions exist within the central region with sizes up to $100 \mathrm{pc}$, and therefore thermal free-free absorption is expected. Recently, Adebahr et al. (2013) observed a spectral flattening due to free-free absorption at $350 \mathrm{MHz}$ in the core region of M82 where intense star formation is known to be occurring. Future observations of M82 at lower frequencies, specifically with LOFAR LBA, may show that this region becomes opaque.

\section{Total magnetic field of M 51}

The total magnetic field strength of M 51 can be determined from the synchrotron emission by assuming equipartition between the energy densities of cosmic rays and magnetic field, using the revised formula of Beck \& Krause (2005). The total magnetic field strength scales with the synchrotron intensity $I_{\text {syn }}$ as:

$B_{\mathrm{tot}, \perp} \propto I_{\mathrm{syn}}^{1 /(3-\alpha)}$,

where $B_{\text {tot, } \perp}$ is the strength of the total field perpendicular to the line of sight. Further assumptions are required on the synchrotron spectral index of $\alpha=-0.8$ and the effective pathlength through the source of $1000 \mathrm{pc} / \cos (i)=1064 \mathrm{pc}$. We also assumed that the polarised emission emerges from ordered fields with all possible inclinations. Here we assume a ratio of CR proton to electron number densities of $K_{0}=100$, which is a reasonable assumption in the star forming regions in the disk (Bell 1978b). Large uncertainties for the pathlength and $K_{0}$ of a factor of 2 would effect the result only by $20 \%$. The effect of adjusting $\alpha$ to between 0.7 and 0.9 produces an error of $5 \%$ in magnetic field strength.

Using these assumptions, we created an image of the total magnetic field in M51 shown in Fig. 9. From this image we also created a radial profile of the total magnetic field strength, shown in Fig. 10. From these figures it is seen that the central region has a total magnetic field strength of between 20 and $30 \mu \mathrm{G}$, the spiral arms of 10 to $20 \mu \mathrm{G}$ and the interarm regions of between 10 and $15 \mu \mathrm{G}$. At regions $r \sim 10 \mathrm{kpc}$ we observe field strengths of $\sim 10 \mu \mathrm{G}$. These values are lower than the values found in Fletcher et al. (2011), who performed a crude separation of thermal and nonthermal synchrotron emission components and derived a synchrotron image to compute the magnetic field strengths. The thermal component is much smaller at $151 \mathrm{MHz}$ than at $4.8 \mathrm{GHz}$, so we expect a smaller error in our estimates. These values are consistent with those modelled by Shneider et al. (2014).

However, errors of the equipartition estimates will become significant in regions away from the CR sources, especially in the outer disk where the observed factor $K$ will increase because energy losses of the CREs are more severe than those of CR protons. Assuming equipartition underestimates the total magnetic field by a factor of $\left(K / K_{0}\right)^{1 / 4}$ in these regions (Beck \& Krause 2005). Fortunately, energy losses are generally weaker at low frequencies and therefore our equipartition estimate should be more accurate than higher frequency estimates.

\section{Cosmic ray propagation in M 51}

\subsection{Radial scale lengths of the total radio emission}

The total extent of disk emission is insufficient as a measure of how far the disk extends as we are limited by the sensitivity of our observations. Therefore, we use the scale length $l$ which describes the emission along the disk as an exponential function, that is, $I_{v} \propto \exp (-r / l)$, where $r$ is the galactocentric distance.

The radial profile of M 51 was taken from the mean in concentric rings with the position angle of the major axis and the inclination of the galaxy taken into account using the values from Table 1. Surrounding background point sources were removed by fitting Gaussians before measuring the radial profile. Several background point sources located in the disk were blanked out. The same was also done to the VLA $(\mathrm{C}+\mathrm{D}$ arrays $) 1.4 \mathrm{GHz}$ image, rescaled to the RBC flux scale from Fletcher et al. (2011), which was placed onto the same grid and resolution as 
D. D. Mulcahy et al.: The nature of the low-frequency emission of M 51

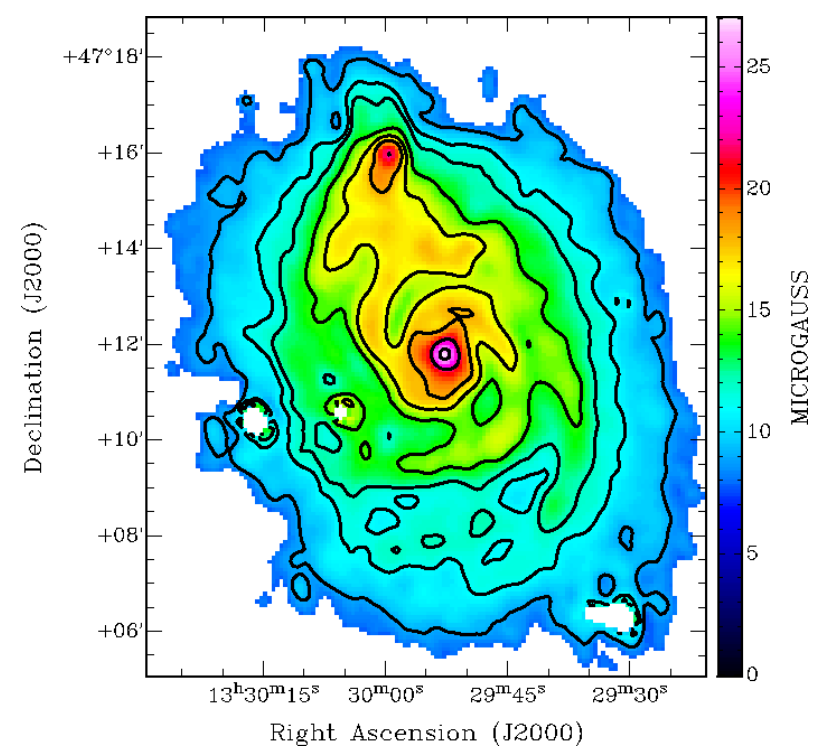

Fig. 9. Total magnetic field of M 51 in $\mu \mathrm{G}$ reaching up to $r \sim 10 \mathrm{kpc}$, determined by assuming energy equipartition. Contours are in levels of $9,11,12,14,16,18,22$ and $26 \mu \mathrm{G}$.

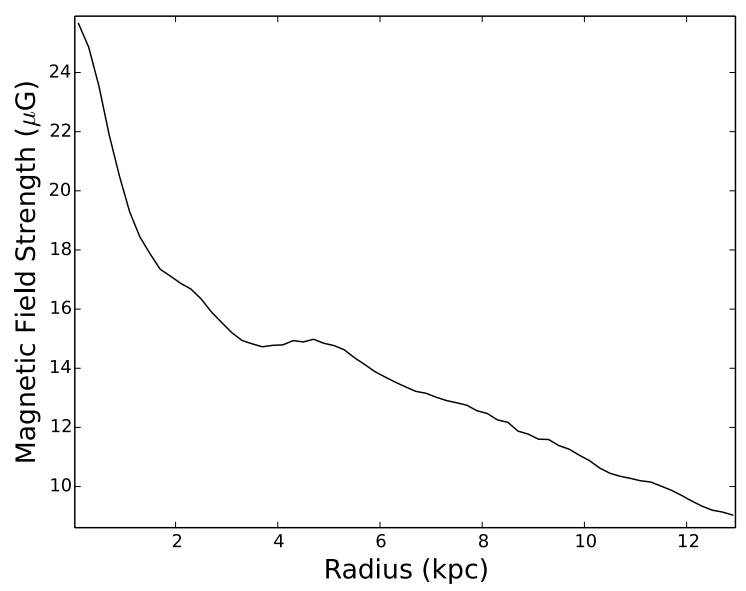

Fig. 10. Radial profile of the total magnetic field of M 51 in $\mu \mathrm{G}$ reaching out to $12 \mathrm{kpc}$ radius.

the $151 \mathrm{MHz}$ image. The interarm region of M 51 appears in the radial profile at $3.3 \mathrm{kpc}$ radius at both frequencies, but is less prominent at $151 \mathrm{MHz}$.

A single exponential profile as fitted by Dumas et al. (2011) is not sufficient, because a break occurs at around $10 \mathrm{kpc}$ at both frequencies (Fig. 11), just beyond the break in the distribution of $\mathrm{H}_{2}$ in M 51 (see Fig. 4 in Schuster et al. 2007). A break in the radial profile of M 51 was also detected in H I at a radius of 9-10 kpc from the galactic centre by Bigiel et al. (2010) (see Table 4).

It is very unlikely that the lack of short spacings can cause the break in the radial profile of M51. LOFAR has excellent inner uv coverage and is sensitive to structures over a half of a degree. The same holds for the $1.4 \mathrm{GHz}$ VLA observations; in $\mathrm{D}$ configuration it is sensitive to structures up to $15^{\prime}$.

Two separate exponential functions were fitted for the inner and outer disk for both images:

$I(R)= \begin{cases}I_{0} \exp \left(-r / l_{\text {inner }}\right) & r \leq 10 \mathrm{kpc} \\ I_{10} \exp \left(-r / l_{\text {outer }}\right) & r \geq 10 \mathrm{kpc} .\end{cases}$

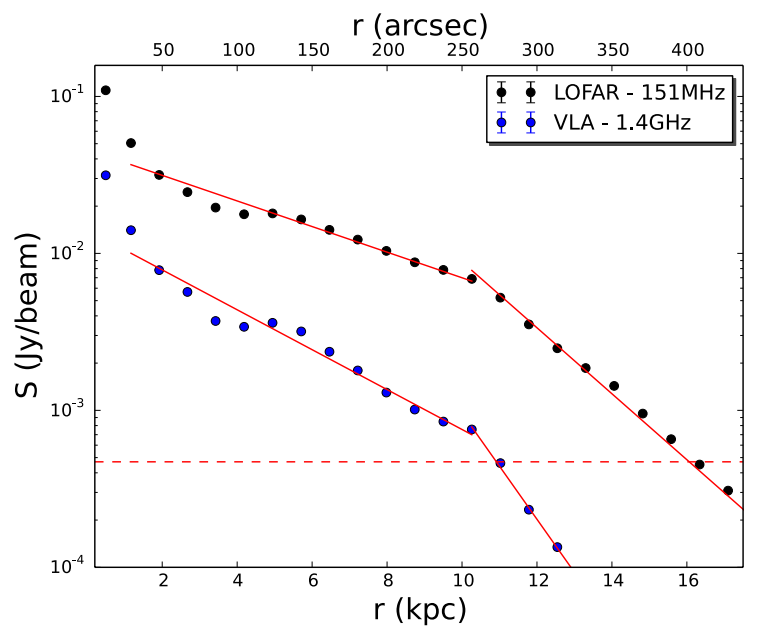

Fig. 11. Radial profile of M 51 at $151 \mathrm{MHz}$ and $1.4 \mathrm{GHz}$. The horizontal red line shows the sensitivity limit $(3 \sigma)$ of the $151 \mathrm{MHz}$ image. Errors are too small to be seen at this scale.

Table 4. Scale lengths of the inner and outer disk of M 51.

\begin{tabular}{lcc}
\hline \hline$v(\mathrm{MHz})$ & $l_{\text {inner }}(\mathrm{kpc})$ & $l_{\text {outer }}(\mathrm{kpc})$ \\
\hline 1400 & $3.4 \pm 0.2$ & $1.28 \pm 0.02$ \\
151 & $5.32 \pm 0.4$ & $2.06 \pm 0.06$ \\
$\mathrm{HI}^{*}$ & 5.5 & $2.1^{*}$ \\
\hline
\end{tabular}

Notes. ${ }^{(*)}$ Estimated from Fig. 2 in Bigiel et al. (2010).

The radial profiles of the continuum emission at $151 \mathrm{MHz}$ and 1.4 GHz with the fitted functions are shown in Fig. 11. The obtained scale lengths for the inner and outer parts of the galaxy are given in Table 4 . The scale length at $1.4 \mathrm{GHz}$ in the inner disk derived by Dumas et al. (2011) of $4.2 \pm 0.5 \mathrm{kpc}$ (scaled to the distance used in this paper) is somewhat consistent with the value derived here $(3.4 \pm 0.2 \mathrm{kpc})$.

The scale length in the outer disk is 2.6 times smaller than the inner scale length at both frequencies. A similar result has been obtained in M 33 (Tabatabaei et al. 2007c) where two exponential scale lengths were fitted. The scale length for $r<4 \mathrm{kpc}$ in M33 is twice as large as the scale length $r>4 \mathrm{kpc}$.

The scale length of radio synchrotron emission is determined by the radial profile of the total magnetic field, distribution of CRE sources and CRE diffusion. The reason for the break is probably due to the break in distribution of CRE sources, closely related to the $\mathrm{H}_{2}$ distribution (see Fig. 4 in Schuster et al. 2007), at about 6-7 kpc, but shifted outwards by a few kpc due to CRE diffusion (see Sect. 6.2).

The scale lengths in the inner and outer disk are larger at $151 \mathrm{MHz}$ than at $1.4 \mathrm{GHz}$ by a factor of $1.6 \pm 0.1$, due to the fact that CRE energy loss processes are weaker at lower frequencies, the CRE lifetime is larger, and hence the radial propagation length of CREs is larger, leading to a larger scale length.

We investigate two models of CRE propagation (Tabatabaei et al. 2013):

(A) Diffusion: the CRE propagation length depends on the average perpendicular and parallel diffusion coefficient, $D$, and the CRE lifetime, $\tau_{\mathrm{CRE}}$, as $l_{\mathrm{dif}} \propto\left(D \tau_{\mathrm{CRE}}\right)^{1 / 2} . D$ may depend weakly on the energy $E$ of the electrons for $E>$ $4 \mathrm{GeV}$ (Engelmann et al. 1990). In the strong field of M51 $\left(B_{\mathrm{tot}} \simeq 15 \mu \mathrm{G}\right)$, we trace electrons of 2.4 and $0.8 \mathrm{GeV}$ at 1400 and $151 \mathrm{MHz}$, respectively, so that the energy dependence of $D$ 

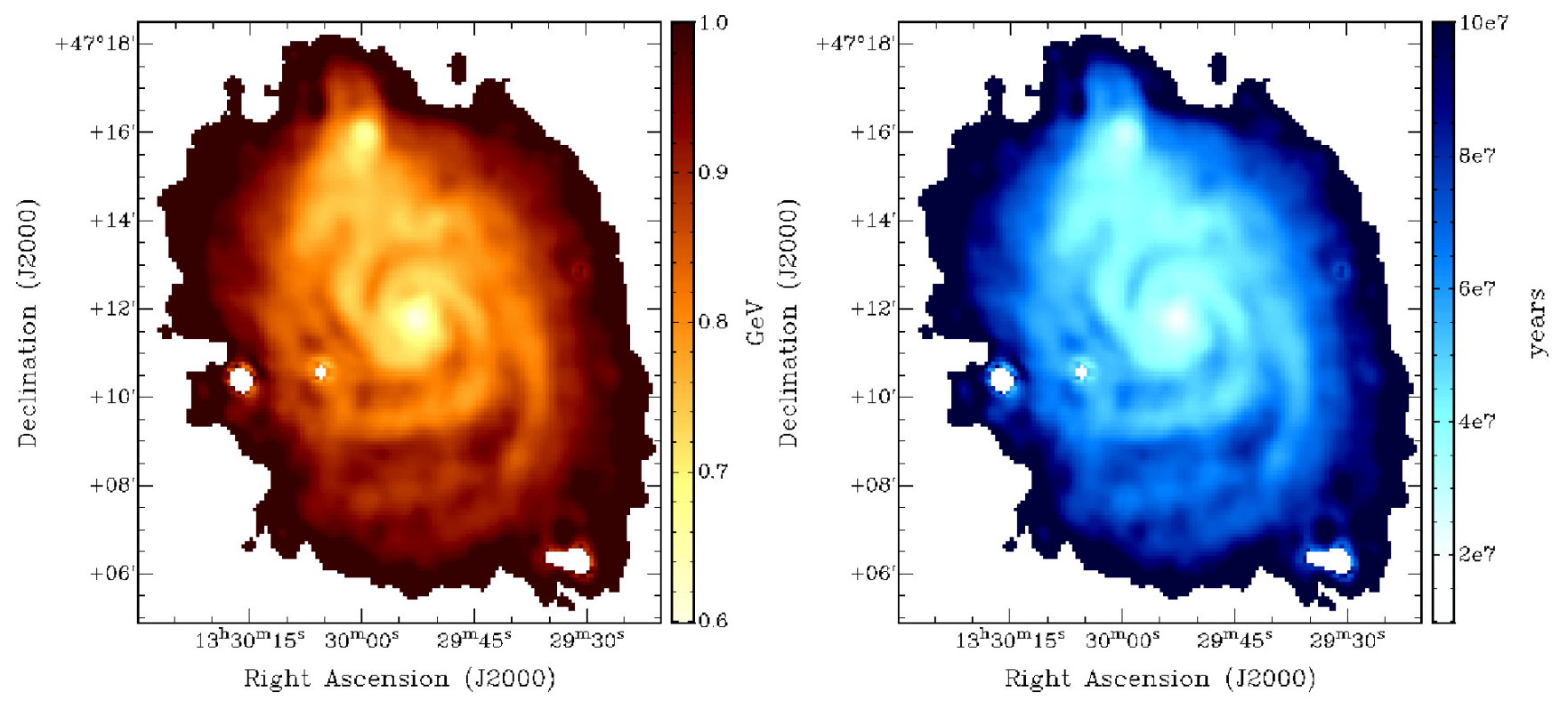

Fig. 12. Images of CRE energy (left) and synchrotron lifetime (right) in M 51.

can be neglected here. As the lifetime of CRE is limited by synchrotron losses (Sect. 6.2, Eq. (7)), $\tau_{\mathrm{CRE}}=\tau_{\text {syn }} \propto B_{\mathrm{tot}}^{-2} E^{-1} \propto$ $B_{\text {tot }}^{-3 / 2} v^{-1 / 2}$, where $v$ is the observation frequency. Insertion into the above relation gives:

$l_{\text {dif }} \propto B_{\text {tot }}^{-3 / 4} v^{-1 / 4}$.

With a frequency ratio of 9.27 , the ratio of propagation lengths is $1 / 1.74$.

(B) Streaming: the CRE propagation length along the magnetic field in case of the streaming instability (Kulsrud \& Pearce 1969) depends on Alfvén velocity $v_{\mathrm{A}} \propto B_{\text {tot }}$ and CRE synchrotron lifetime $\tau_{\text {syn }}$ :

$l_{\text {stream }}=v_{\mathrm{A}} \tau_{\mathrm{CRE}} \propto B_{\text {tot }}^{-1 / 2} v^{-1 / 2}$.

With a frequency ratio of 9.27 , the ratio of propagation lengths is $1 / 3.04$.

The ratios of scale lengths observed at 1400 and $151 \mathrm{MHz}$ of about 1/1.6 (Table 4) agree with the diffusion model.

Both models are rather simplistic and should only be used to give tendencies about the intrinsic distribution and the magnitude of the CRE propagation length. A more detailed analysis needs a proper description of CRE sources, magnetic field distribution, CRE loss processes and CRE propagation mechanisms.

\subsection{CRE diffusion coefficients in M51}

CREs lose their energies via a number of different processes such as synchrotron radiation, inverse Compton (IC) radiation, non-thermal bremsstrahlung, ionisation, and adiabatic expansion. Out of these processes, synchrotron and inverse Compton losses have the same dependence on particle energy and therefore are difficult to distinguish from the radio spectrum alone. Inverse Compton loss in the galaxy's radiation field is generally smaller than synchrotron loss in galaxies (Heesen et al. 2014) and is neglected in the following.

We use the equations from Pohl \& Schlickeiser (1990) to calculate the CRE energy $E$ and the synchrotron lifetime throughout M 51 (Fig. 12):

$\left(\frac{E}{\mathrm{GeV}}\right)=\left(\frac{v}{16.1 \mathrm{MHz}}\right)^{\frac{1}{2}}\left(\frac{B_{\mathrm{tot}, \perp}}{\mu \mathrm{G}}\right)^{-\frac{1}{2}}$ and

$$
\left(\frac{\tau_{\mathrm{syn}}}{\mathrm{yr}}\right)=8.352 \times 10^{9}\left(\frac{E}{\mathrm{GeV}}\right)^{-1}\left(\frac{B_{\mathrm{tot}}}{\mu \mathrm{G}}\right)^{-2} .
$$

If we take $l_{\text {dif }}=1.45 \mathrm{kpc}$ from Sect. 7 as the typical distance that a CRE travels from its origin, the diffusion coefficient $\mathrm{D}$ of the electrons can be estimated by (see e.g. Istomin 2014):

$$
D=\frac{l_{\mathrm{dif}}^{2}}{4 \tau_{\mathrm{syn}}}
$$

With an average synchrotron lifetime of $\simeq 4.8 \times 10^{7}$ years across the star forming region of the galaxy, we estimate $D \simeq 3.3 \times$ $10^{27} \mathrm{~cm}^{2} \mathrm{~s}^{-1}$. This value is smaller than that of Fletcher et al. (2011) who disregarded the factor of 4 in Eq. (8) and assumed $l_{\text {dif }}=1 \mathrm{kpc}$ at $5 \mathrm{GHz}$ which seems too high compared to our results. Tabatabaei et al. (2013) estimated $l_{\text {dif }}>0.5 \mathrm{kpc}$ in M 51 at $1.4 \mathrm{GHz}$ where $\tau_{\text {syn }} \simeq 1.5 \times 10^{7}$ years, which yields $D>1.3 \times 10^{27} \mathrm{~cm}^{2} \mathrm{~s}^{-1}$, consistent with our result. On the other hand, the values of $D \sim(2-4) \times 10^{28} \mathrm{~cm}^{2} \mathrm{~s}^{-1}$ used in the models by Moskalenko \& Strong (1998) for the Milky Way are higher. It is important to note that the diffusion coefficient may vary with magnetic field strength and degree of field order. As M51 has a stronger and possibly more turbulent magnetic field than the Milky Way, the diffusion coefficient in M 51 could be lower.

Figure 12 shows us that at the edge of the extended disk $r \sim 10 \mathrm{kpc}$, the synchrotron lifetime for CREs is found to be approximately around $10^{8} \mathrm{yr}<\tau_{\text {syn }} \leq 2 \times 10^{8} \mathrm{yr}$. Using a diffusion coefficient of $\sim 3.3 \times 10^{27} \mathrm{~cm}^{2} \mathrm{~s}^{-1}$ and Eq. (8), the electrons at $r \sim 10 \mathrm{kpc}$ are able to travel up to $2.1-3.0 \mathrm{kpc}$, neglecting other energy losses. This takes us out to the edge of the extended disk detected with LOFAR.

\subsection{Diffusion of CREs into the interarm regions}

According to observations of several spiral galaxies such as NGC 6946 with the GMRT at $333 \mathrm{MHz}$, the arm and interarm regions are indiscernible (Basu et al. 2012a), while in the case of M51 at $151 \mathrm{MHz}$ the arm and interarm regions are clearly separated. This is unexpected because at $151 \mathrm{MHz}$ emission 

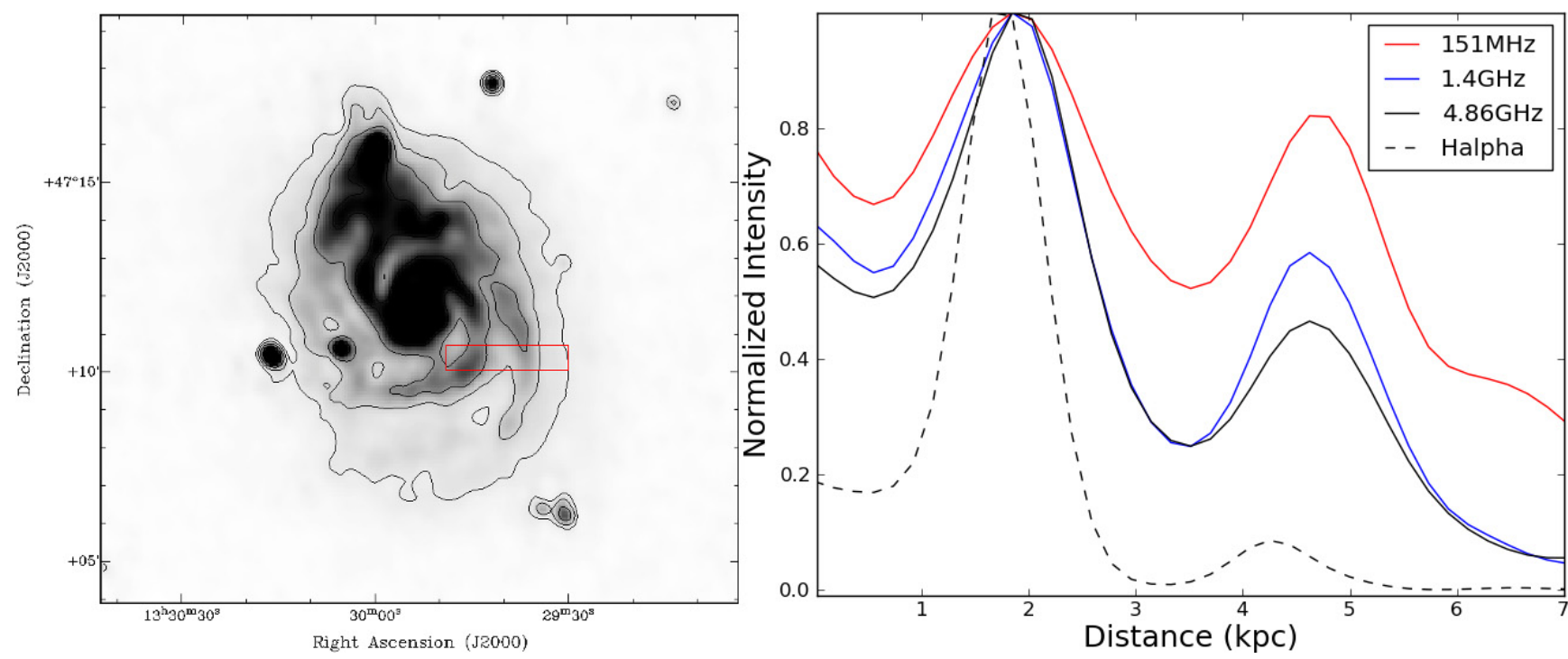

Fig. 13. Intensity profile showing the arm and interarm contrasts of $\mathrm{H} \alpha$ and three different radio frequencies along a slice through $\mathrm{M} 51$. The location of this slice is shown in the image to the right. The peak flux densities from each image are normalised to 1.

emerges from an even older population of CREs that should diffuse further away from star forming regions in the spiral arms.

To quantify our result, we used a $\mathrm{H} \alpha$ image (Greenawalt et al. 1998) and the $4.86 \mathrm{GHz}$ and $1.4 \mathrm{GHz}$ VLA images from Fletcher et al. (2011) in addition to our LOFAR $151 \mathrm{MHz}$ image. All images were smoothed to a common resolution of $20^{\prime \prime}$ and transformed to the same grid. Figure 13 shows a slice of M 51 for all 4 images through a region where the arm-interarm contrast could be best studied. The slice is taken at a fixed declination of $+47^{\circ} 10^{\prime} 23^{\prime \prime}$ and extends from a right ascension of $13^{\mathrm{h}} 29^{\mathrm{m}} 30.1^{\mathrm{s}}$ to $13^{\mathrm{h}} 29^{\mathrm{m}} 49.2^{\mathrm{s}}$.

The $4.86 \mathrm{GHz}$ and $1.4 \mathrm{GHz}$ profiles are very similar, indicating that the CREs radiating at $1.4 \mathrm{GHz}$, which have about half of the energy, are not diffusing much further than at the $4.86 \mathrm{GHz}$ eletrons. At $151 \mathrm{MHz}$, tracing CREs with 3 times less energy compared to $1.4 \mathrm{GHz}$, a much smoother gradient indicates that there are considerably more CREs diffusing far into the interarm region.

While we see considerable CRE diffusion into the interarm region of M 51, the question arises why we do not observe CREs diffusing the same distances as in the $333 \mathrm{MHz}$ images of other galaxies by Basu et al. (2012a). One reason could be a stronger and more turbulent magnetic field in the spiral arms of M51 compared to galaxies like NGC 6946. The shorter synchrotron lifetime (Eq. (7)) leads to a shorter propagation length, making it harder to diffuse into the interarm regions.

\section{Wavelet cross-correlation and CRE propagation length}

Wavelet transforms have been applied to several images of galaxies, for example M 33 (Tabatabaei et al. 2007a) and M 51 (Dumas et al. 2011). Wavelet transforms and cross-correlations allow us to separate the diffuse emission components from compact sources and to compare the emission at different wavelengths which we shall perform in this section. It is also useful when studying the radio (FIR) correlation at various spatial scales, especially when separating the differences of the correlation between the arm and interarm regions (see for example Dumas et al. 2011 and Basu et al. 2012b).

The wavelet coefficient is defined as:

$W(a, \boldsymbol{x})=\frac{1}{a^{\kappa}} \int_{-\infty}^{+\infty} f\left(\mathbf{x}^{\prime}\right) \psi^{*}\left(\frac{\mathbf{x}^{\prime}-\boldsymbol{x}}{a}\right) \mathrm{d} \mathbf{x}^{\prime}$,

where $\psi(\boldsymbol{x})$ is the analysing wavelet, $\boldsymbol{x}=(x, y), f(\boldsymbol{x})$ is a twodimensional function which in this case is an image, $a$ and $\kappa$ are the scale and the normalisation parameters, respectively, and finally the ${ }^{*}$ symbol denotes the complex conjugate.

The Mexican Hat wavelet is a real isotropic wavelet with a minimal number of oscillations and was used as we wish to have more independent points:

$\psi(\rho)=\left(2-\rho^{2}\right) \mathrm{e}^{-\rho^{2} / 2}$.

Our LOFAR image at $151 \mathrm{MHz}$, the VLA image at $1.4 \mathrm{GHz}$ and the $70 \mu \mathrm{m}$ image from the Multiband Imaging Photometer for Spitzer (Spitzer-MIPS; Rieke et al. 2004) (courtesy of Tabatabaei) were used for this analysis. FIR flux densities from normal galaxies are commonly taken to indicate the rate of recent star formation. Hinz et al. (2004) found that FIR emission at 24 and $70 \mu \mathrm{m}$ follows closely the structure of the ionised gas in M33, indicating that it is heated mostly by hot ionising stars.

All images were made to have the same size, be on the same grid and were smoothed to a PSF with FWHM of $20^{\prime \prime}$. Bright background sources from the VLA and LOFAR images were subtracted from the images. The images have been decomposed into 10 different scales $a$ with log spacing in order to compare the morphology between the three different images (Fig. 14).

The individual HII regions within the spiral arms in the Spitzer and the VLA images coincide quite well at the smallest scale $(a=754 \mathrm{pc})$. However, these regions are not visible as well in the LOFAR image at the same scale. The spiral arms are prominent at all three wavelengths at scales up to $1080 \mathrm{pc}$. The spiral arms become indiscernible at a scale of $2212 \mathrm{pc}$, while in the LOFAR image, and to a smaller extent in the VLA image, the southern spiral arm can be seen to extend much farther out.

At a scale of $4440 \mathrm{pc}$, no discernible features can be seen at any of the wavelengths, only the underlying diffuse disk with 


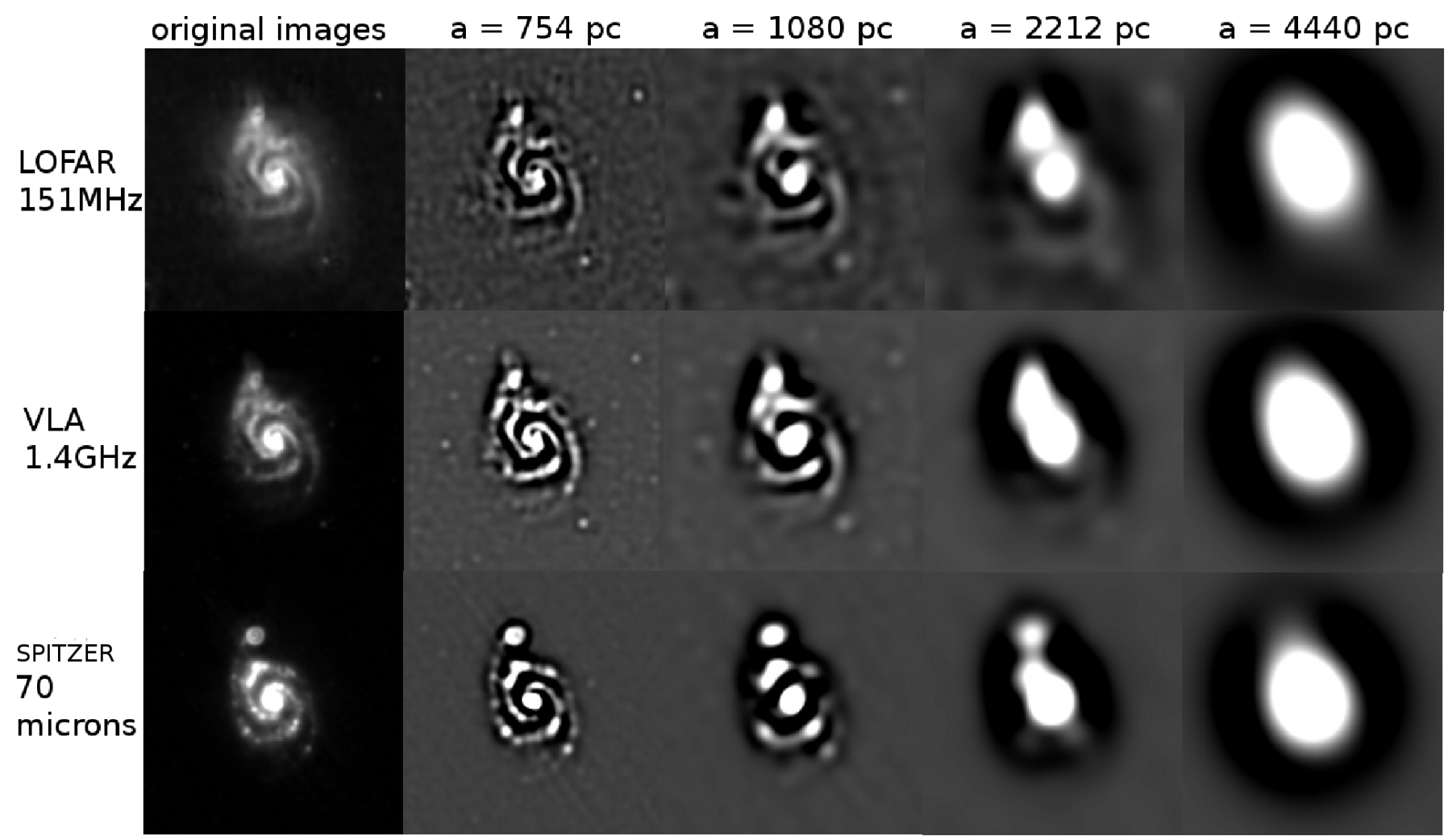

Fig. 14. Wavelet decomposition of the three available datasets ( $151 \mathrm{MHz}, 1.4 \mathrm{MHz}$ and $70 \mu \mathrm{m}$ ) at four different spatial scales. The LOFAR $151 \mathrm{MHz}$ emission clearly extends farther out.

a gentle radial decrease in intensity. It should be noted that the radial decrease is slower for M 51 in the LOFAR dataset, consistent with Fig. 11.

The wavelet cross-correlation is a useful method to compare different images as a function of spatial scales (Frick et al. 2001; Dumas et al. 2011; and Tabatabaei et al. 2007a). Whilst normal cross-correlation analysis such as pixel to pixel correlation can be dominated by bright extended regions or large scale structure, the wavelet cross-correlation allows the analysis of a scale-dependent correlation between two images.

The cross-correlation coefficient at scale $a$ is defined as:

$r_{w}(a)=\frac{\iint W_{1}(a, \boldsymbol{x}) W_{2}^{*}(a, \boldsymbol{x}) \mathrm{d} \boldsymbol{x}}{\left[M_{1}(a) M_{2}(a)\right]^{1 / 2}}$

where $M(a)$ is the wavelet spectrum:

$M(a)=\int_{-\infty}^{+\infty} \int_{-\infty}^{+\infty}|W(a, x)|^{2} \mathrm{~d} \boldsymbol{x}$.

The value of $r_{w}$ can range between -1 (total anticorrelation) and +1 (total correlation). Plotting $r_{w}$ against scale shows how well structures at different scales cross-correlate in intensity and location between the two images (Fig. 15).

The cross-correlation spectrum between the $1.4 \mathrm{GHz}$ and $151 \mathrm{MHz}$ images is extremely good for all scales, except for scales less than $1 \mathrm{kpc}$ (Fig. 15). Overall, $r_{\mathrm{w}}$ for $151 \mathrm{MHz}-70 \mu \mathrm{m}$ is smaller compared to $1.4 \mathrm{GHz}-70 \mu \mathrm{m}$.

The break scale at which $r_{\mathrm{w}}=0.75$ is reached for a cross-correlation spectrum between radio synchrotron and FIR emission is taken as a measure of the diffusion length $l_{\text {dif }}{ }^{5}$,

\footnotetext{
5 The absolute values of $l_{\text {dif }}$ depend on the definition of the break scale. $r_{\mathrm{w}}=0.50$ was used by Tabatabaei et al. (2013) to define the break scale because the correlations analysed in that paper were generally weaker than the ones in this paper.
}

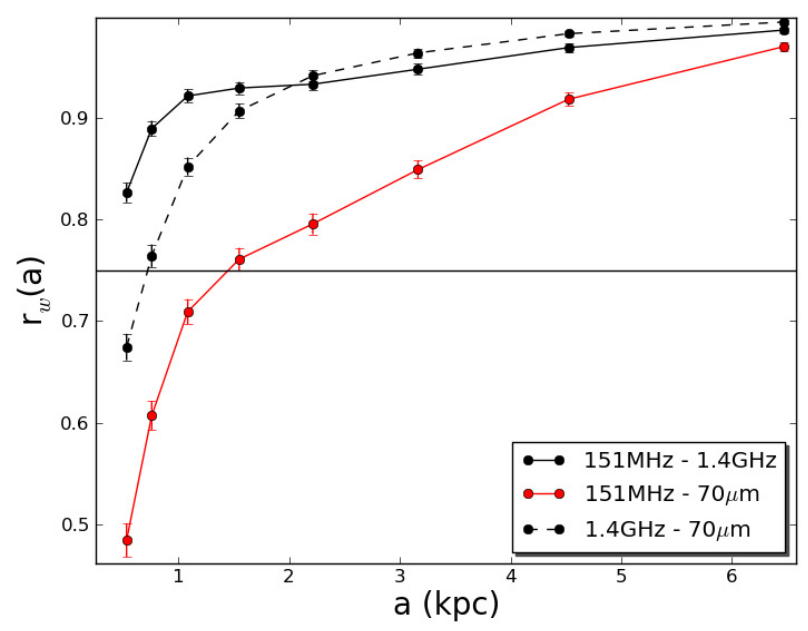

Fig. 15. Wavelet cross-correlation spectra between the three data sets. The correlation coefficient of $r_{w}=0.75$ defining the break scale is shown by the solid line.

which depends on the diffusion coefficient and CRE lifetime as $l_{\text {dif }}=2\left(D \tau_{\mathrm{CRE}}\right)^{0.5}$ (see Sect. 6.1).

From Fig. 15 we derive that $l_{\text {dif }}$ is approximately 720 pc for the cross-correlation between $1.4 \mathrm{GHz}$ and $70 \mu \mathrm{m}$ and about $1.45 \mathrm{kpc}$ between $151 \mathrm{MHz}$ and $70 \mu \mathrm{m}$. This is interpreted as the lower-energy CREs propagating from the star forming regions have longer lifetimes and travel further.

The ratio of propagation lengths of 2.0 is consistent with diffusive CRE propagation (Eq. (4)).

Dumas et al. (2011, their Fig. 7) derived wavelet crosscorrelation spectra only between the total radio and $24 \mu \mathrm{m}$ data which cannot be directly compared with the results in this paper. 
Still, a trend of $l_{\text {dif }}$ increasing with decreasing frequency is also seen.

Our value for $l_{\text {dif }}$ in M 51 between $1.4 \mathrm{GHz}$ and $70 \mu \mathrm{m}$ is smaller than that for $\mathrm{M} 31$ ( $l_{\text {dif }}=3 \pm 1 \mathrm{kpc}$ for our definition of the break scale) and also for NGC 6946 ( $\left.l_{\text {dif }}=5 \pm 1 \mathrm{kpc}\right)$ (Tabatabaei et al. 2013). This further supports our result from Sect. 6.3 that the typical propagation length of CREs in M 51 is small due to their short synchrotron lifetime in the strong field. This also answers the question why the arm and interarm contrast is still seen in M 51 at $151 \mathrm{MHz}$ but not in NGC 6946 at $333 \mathrm{MHz}$ (Basu et al. 2012a).

M 51 and M 31 have a similar degrees of magnetic field order, so that the relation between $l_{\mathrm{dif}}$ and the degree of field order proposed by Tabatabaei et al. (2013) predicts similar values of $l_{\text {dif }}$. However, we find a value only half as large in M 51 . Tabatabaei et al. (2013) assumed that the CRE lifetime is given by the energy-independent confinement time of about $3 \times 10^{7} \mathrm{yr}$ within the disk that is limited by escape loss, while in M 51 the CRE lifetime is limited by the shorter synchrotron lifetime (see Fig. 12 right).

\section{Detection of polarised sources}

\subsection{Introduction}

Previously, very little has been done to study polarisation at frequencies below $200 \mathrm{MHz}$, and polarisation characteristics cannot be extrapolated directly from higher frequencies. From previous observations at $350 \mathrm{MHz}$ (Schnitzeler et al. 2009), one would optimistically expect to detect one polarised source with a polarisation degree of a few percent for every four square degrees on the sky (Bernardi et al. 2013). It is worth noting that for the observations of Schnitzeler et al. (2009) at $350 \mathrm{MHz}$, the resolution was $2.7 \times 4.7$ arcmin with a noise of polarised intensity of $0.5 \mathrm{mJy} /$ beam.

Studies of polarisation at frequencies below $300 \mathrm{MHz}$ are now becoming possible, partly due to the construction of LOFAR (van Haarlem et al. 2013) and the Murchison Widefield Array (MWA; Lonsdale et al. 2009), and to the introduction of new techniques to analyse polarisation, in particular RM synthesis (Brentjens \& de Bruyn 2005). Recently, Bernardi et al. (2013) performed a 2400 square degree polarisation survey at $189 \mathrm{MHz}$ with the MWA with a 7' beam. Out of a catalogue of 137 sources brighter than 4 Jy in flux density, only one source was detected in polarisation. Both beam depolarisation and internal Faraday dispersion could reduce the measured polarised emission, therefore higher resolution observations are essential. Gießübel et al. (2013), whilst detecting diffuse polarisation in a nearby galaxy namely M31 - for the first time below $1 \mathrm{GHz}$, also created a catalogue of 33 polarised background sources. Extrapolation of these results to the frequencies of LOFAR would result in very few source detections and no detections of polarised diffuse emission from the star forming disks of galaxies. However, these observations are limited by the large angular resolution of $4^{\prime}$. It needs to be seen if beam depolarisation plays a major role. Farnes et al. (2013) applied RM Synthesis to data from the Giant Meterwave Radio Telescope (GMRT) at $610 \mathrm{MHz}$ and found that M 51 is depolarised to below the sensitivity limit.

LOFAR is the perfect instrument to measure Faraday depths with a high precision and hence should allow us to detect weak magnetic fields and low electron densities that are unobservable at higher frequencies. Importantly, LOFAR with its high sensitivity and high angular resolution should be able to reduce depolarisation effects.
LOFAR has already produced polarisation results: Iacobelli et al. (2013) were able to detect a faint and morphologically complex polarised foreground of the highly polarised Fan region, in agreement with previous WSRT observations (Bernardi et al. 2009).

\subsection{Applying RM synthesis}

For each frequency channel, Stokes $Q$ and $U$ were imaged at $20^{\prime \prime}$ resolution with an area of 17.3 square degrees with CASA. Therefore no primary beam correction was applied, which leads to errors. However, using AWimager for a field of this size and at this high resolution for every frequency channel would require much more processing time and power than available. Each channel image was inspected and all channels with RFI contamination were discarded. This resulted in 3774 final images.

Rotation measure (RM) synthesis was performed on these images using software written by the LOFAR Magnetism Key Science Project (MKSP). The rms noise for the uncorrected primary beam image for polarised intensity was found to be $100 \mu \mathrm{Jy} /$ beam/rmsf. For Stokes $U$ and $Q$, the rms noise was found to be $123 \mu \mathrm{Jy} /$ beam and $132 \mu \mathrm{Jy} / \mathrm{beam}$, respectively. The resulting Faraday cube at $20^{\prime \prime}$ resolution was then cleaned, using the RM Clean code of Heald et al. (2009) for MIRIAD (Sault et al. 1995). A maximum of 1000 iterations was used as well as a $1 \sigma$ cutoff level.

Usually, instrumental polarisation is located at a Faraday depth of $0 \mathrm{rad} \mathrm{m}^{-2}$. However, due to the ionospheric RM correction that was performed, this instrumental polarisation is shifted by the average RM correction applied to the data. Therefore, for this observation, the instrumental polarisation is located at a Faraday depth of approximately $-1 \mathrm{rad} \mathrm{m}^{-2}$.

The resolution in Faraday depth $\phi$ is given by the measured full width half maximum of the RM spread function (RMSF; Brentjens \& de Bruyn 2005):

$\phi=\frac{2 \sqrt{3}}{\Delta \lambda^{2}}$

where $\Delta \lambda^{2}$ is the width of the observed $\lambda^{2}$ distribution.

A minimum frequency of $115.9 \mathrm{MHz}$ and a maximum of $176 \mathrm{MHz}$ gives us $\phi=0.91 \mathrm{rad} \mathrm{m}^{-2}$. The largest detectable structure in Faraday spectrum (Brentjens \& de Bruyn 2005) is given by:

$\phi_{\max }=\frac{\pi}{\lambda_{\min }^{2}}$.

For our observations $\phi_{\max }=1.085 \mathrm{rad} \mathrm{m}^{-2}$. Any larger structure will be depolarised.

The error in Faraday depth is found by the following expression:

$\Delta \phi=\frac{\phi}{2 S / N}$

where $\phi$ is the FWHM of the RMSF and S/N is the signalto-noise ratio of the peak of the component in the Faraday spectrum.

Following the foreground RM measured by Fletcher et al. (2011), Heald et al. (2009) and Horellou et al. (1992), we would expect the polarised signal of M 51 to be centred on $+9 \mathrm{rad} \mathrm{m}^{-2}$. At low frequencies, the polarised signal of M 51 should be centred around this value, with a dispersion smaller than that in 
Fig. 10 of Fletcher et al. (2011) because only the halo and the foreground contribute to the dispersion.

No obvious diffuse polarisation from M51 could be detected by looking into the FD-cubes. An integration over $25 \mathrm{rad} \mathrm{m}^{-2}$ wide parts in Faraday-space, for the area of M 51, that is above the $1 \mathrm{mJy}$ contour in the total power image, delivered only noise with a standard deviation of $\sim 100 \mu \mathrm{Jy}$. The same integration was carried out for four regions with the same size close to M 51. Those delivered a slightly lower standard deviation of $80 \mu \mathrm{Jy}$. For all integrations the region from $-5 \mathrm{rad} \mathrm{m}^{-2}$ to $+5 \mathrm{rad} \mathrm{m}^{-2}$ was excluded to not include the instrumental peak around $-1 \mathrm{rad} \mathrm{m}^{-2}$. We attribute the slightly higher standard deviation in the M51 field to the remnants of the sidelobes of the instrumental peaks. Therefore we set a $5 \sigma$ detection limit for the integrated polarised intensity of M 51 to $0.5 \mathrm{mJy}$ which corresponds to a polarisation degree of $0.006 \%$.

This is not surprising in view of the small $\phi_{\max }$ and is in line with Farnes et al. (2013) who were unable to detect M 51 in polarisation at $610 \mathrm{MHz}$ with the GMRT.

\subsection{Extragalactic polarised sources}

Taking five times the rms noise of $100 \mu \mathrm{Jy} /$ beam/rmsf as the detection threshold and assuming an average degree of polarisation of $1 \%$, all sources with flux densities above $50 \mathrm{mJy} /$ beam were checked for polarisation, with six detections of polarised background extragalactic radio galaxies in the field. Two of these sources are partly resolved, with polarisation detected in the lobes. Three sources were found to be outside the FWHM of the station beam of 3.6 degrees. This results in a mean detection rate of one polarised source for every 1.7 square degrees. As these sources are several degrees from the phase centre, the primary beam becomes important. To apply the primary beam, the fluxes of these polarised sources were scaled by comparing a primary beam corrected image created from AWimager and the final CASA image. The difference for each source was found between the two images and applied. This corrected flux is shown in the following Faraday spectra and Table 5.

Where data are available from higher frequencies, notably from Taylor et al. (2009), we can determine the depolarisation ratio as defined in Beck (2007):

$\mathrm{DP}(151,1400)=\left(\mathrm{PI}_{151} / \mathrm{PI}_{1400}\right)\left(v_{1400} / v_{151}\right)^{\alpha}$

where $\alpha$ is the synchrotron spectral index and $v_{i}$ is the respective frequency. A value of 1 means no additional depolarisation at $151 \mathrm{MHz}$ with respect to $1400 \mathrm{MHz}$.

In the following, we will only address the resolved sources briefly. A summary of all detections are given in Table 5. The Faraday spectra of the remaining sources can be seen in Fig. 18.

\subsubsection{J133920+464115}

Strong polarisation was detected in this single lobe radio galaxy B3 1337+469, solely from the lobe itself $(J 133920+464115)$ that contains two spatially separated components observable in both total and polarised intensity. The northern component, which is brighter, is detected at a Faraday depth of $+20.5 \pm 0.1 \mathrm{rad} \mathrm{m}^{-2}$, while the southern component has $+20.75 \pm 0.1 \mathrm{rad} \mathrm{m}^{-2}$.

The Faraday spectra and total intensity image for this source are shown in Fig. 16.

This source is only partially resolved in the NVSS and from Taylor et al. (2009) it is polarised in the core with a RM of $22.5 \pm 3.9 \mathrm{rad} \mathrm{m}^{-2}$ and a polarisation degree of $3.66 \pm 0.07 \%$. 

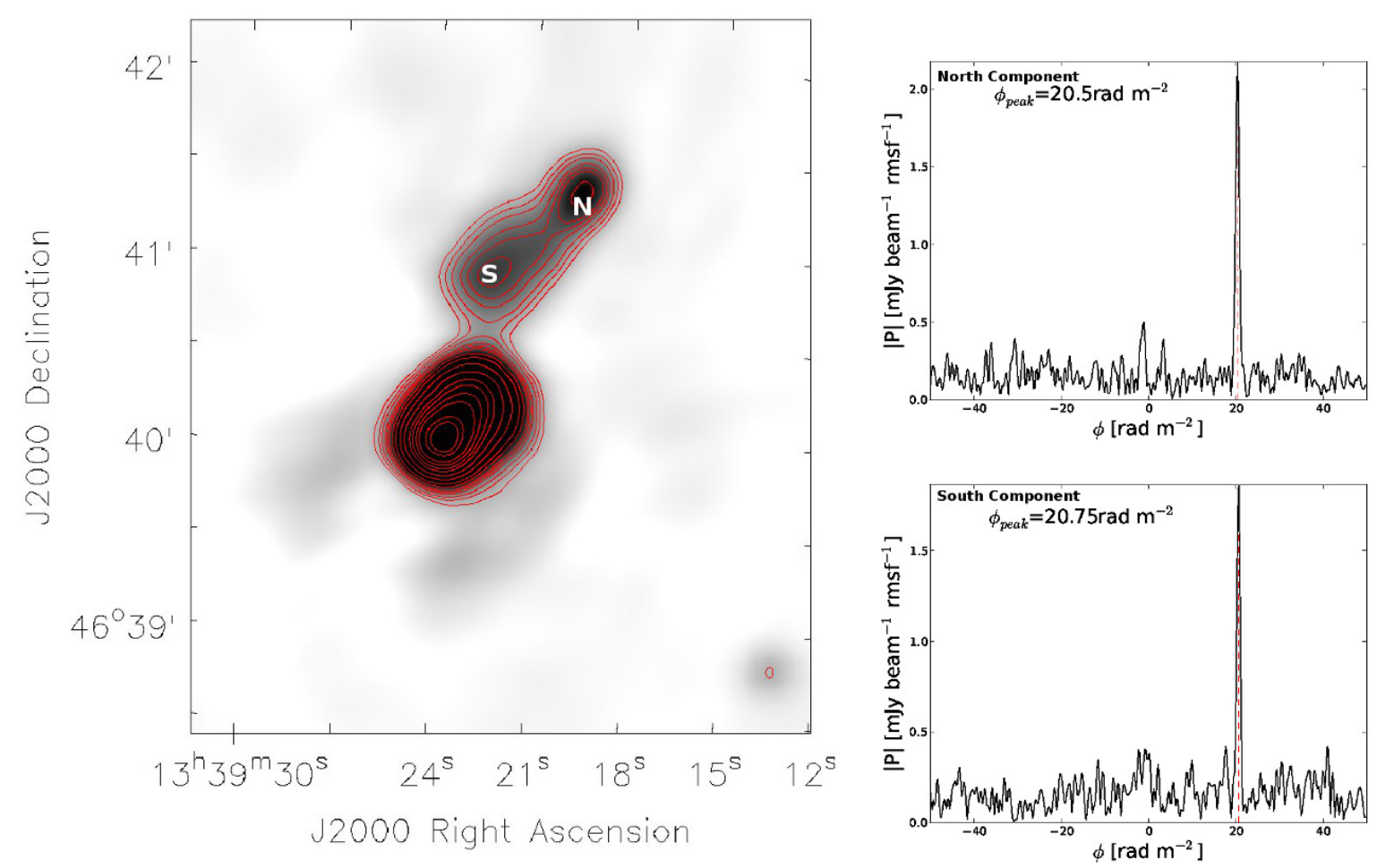

Fig. 16. Radio lobe J133920+464115 seen in total intensity (left) with the two marked spatial components and the corresponding Faraday spectra (right). rmsf is the half-power width of the RM spread function.

This is completely depolarised at $151 \mathrm{MHz}$. At $1.4 \mathrm{GHz}$, the jet component has a RM of $5.5 \pm 7.3 \mathrm{rad} \mathrm{m}^{-2}$, and a polarisation degree of $7.06 \pm 0.23 \%$.

For the north component we do see a hint of a polarisation emission at $+3.5 \pm 0.1 \mathrm{rad} \mathrm{m}^{-2}$ which could correspond to the $\mathrm{RM}$ found in the jet component in Taylor et al. (2009). However, as the source is only partially resolved in the NVSS, it is very difficult to be confident about which RMs correspond to which positions of the source.

Taking the polarised intensity at the Faraday depth $+20.5 \pm$ $0.1 \mathrm{rad} \mathrm{m}^{-2}$ we see that the depolarisation factor for the lobe to be 0.196 .

While the single lobe is somewhat depolarised at $151 \mathrm{MHz}$ compared to the $1.4 \mathrm{GHz}$ value of Taylor et al. (2009), the core is completely depolarised. This suggests that the LaingGarrington effect occurs (Garrington et al. 1988) where the stronger jet is closer to us, is seen through a smaller pathlength of magneto-ionic material, and thus shows less depolarisation.

\subsubsection{J132626+473741}

This source is also a single lobe radio galaxy with a strong polarisation detection in the lobe at $\phi=+3.2 \pm 0.1 \mathrm{rad} \mathrm{m}^{-2}$. Unfortunately, this source was not detected by Taylor et al. (2009) and therefore no comparison can be made with higher frequencies. At the peak of the lobe, the polarisation degree is $2.9 \pm 0.2 \%$, very similar to the previous source J133920+464115, and has one clear component in the Faraday spectrum. Closer to the edge of the radio lobe, marked "O" in Fig. 17, we detect a possible secondary component at $\phi=+19.5 \mathrm{rad} \mathrm{m}^{-2}$ with a polarised flux density of $390 \mu \mathrm{Jy} /$ beam and possibly a third one at $\phi=+30.5 \mathrm{rad} \mathrm{m}^{-2}$ with a polarised flux density of $280 \mu \mathrm{Jy} /$ beam. The Faraday spectra and total intensity image for this source are shown in Fig. 17.

\section{Discussion}

The radial profiles of M51 at $151 \mathrm{MHz}$ and $1.4 \mathrm{GHz}$, which consist of mostly nonthermal synchrotron emission, are fitted by a larger exponential profile at radii $r \leq 10 \mathrm{kpc}$ than at $\geq 10 \mathrm{kpc}$. As mentioned by Tabatabaei et al. (2007c), synchrotron emission mimics the distribution of the star forming regions in the disk. As we showed in this study, this still applies for low frequencies. The sharp break in star formation rate at about $7 \mathrm{kpc}$ radius is also seen in synchrotron emission, but is shifted and flattened at $151 \mathrm{MHz}$ due to the propagation of low-energy CREs. As a result, the expectation that galaxies would be extremely large at low frequencies (Beck et al. 2013) does not seem to hold.

Our observations demonstrate that a significant magnetic field is present up to $16 \mathrm{kpc}$ in the outer disk due to the fact that we observe synchrotron emission. The magnetic field must be at least $3.25 \mu \mathrm{G}$ in order for synchrotron emission to be visible despite inverse Compton losses. However, it is very difficult to determine accurately the magnetic field strength in these regions because of the uncertainty of the CR proton to electron number density in areas of low star formation.

Our results give no indication of a flattening of the integrated spectrum of M 51 down to $151 \mathrm{MHz}$ and therefore disagree with (Pohl et al. 1991; their Fig. 11). Pohl et al. (1991) and Hummel (1991) argued that a steepening in the integrated spectrum of M 51 occurs beyond about $1 \mathrm{GHz}$, caused by the energy losses of the CREs. It is likely that this interpretation was affected by the potential inaccuracies of a few flux density measurements.

According to Pohl et al. (1991), there should be a break $E_{\mathrm{b}}$ of the CRE energy spectrum at energy $E_{\mathrm{b}}$ (and a steepening of the synchrotron spectrum beyond the corresponding frequency according to Eq. (6)):

$E_{\mathrm{b}}=\frac{3.3 \times 10^{15}\left(\frac{\operatorname{div}(v)}{\mathrm{s}^{-1}}\right)+8\left(\frac{n}{1 \mathrm{~cm}^{-3}}\right)}{\left(\frac{w(r)}{0.7 \mathrm{eV} \mathrm{cm}^{-3}}\right)+0.25\left(\frac{B_{\perp}}{3.25 \mu \mathrm{G}}\right)^{2}} \mathrm{GeV}$, 

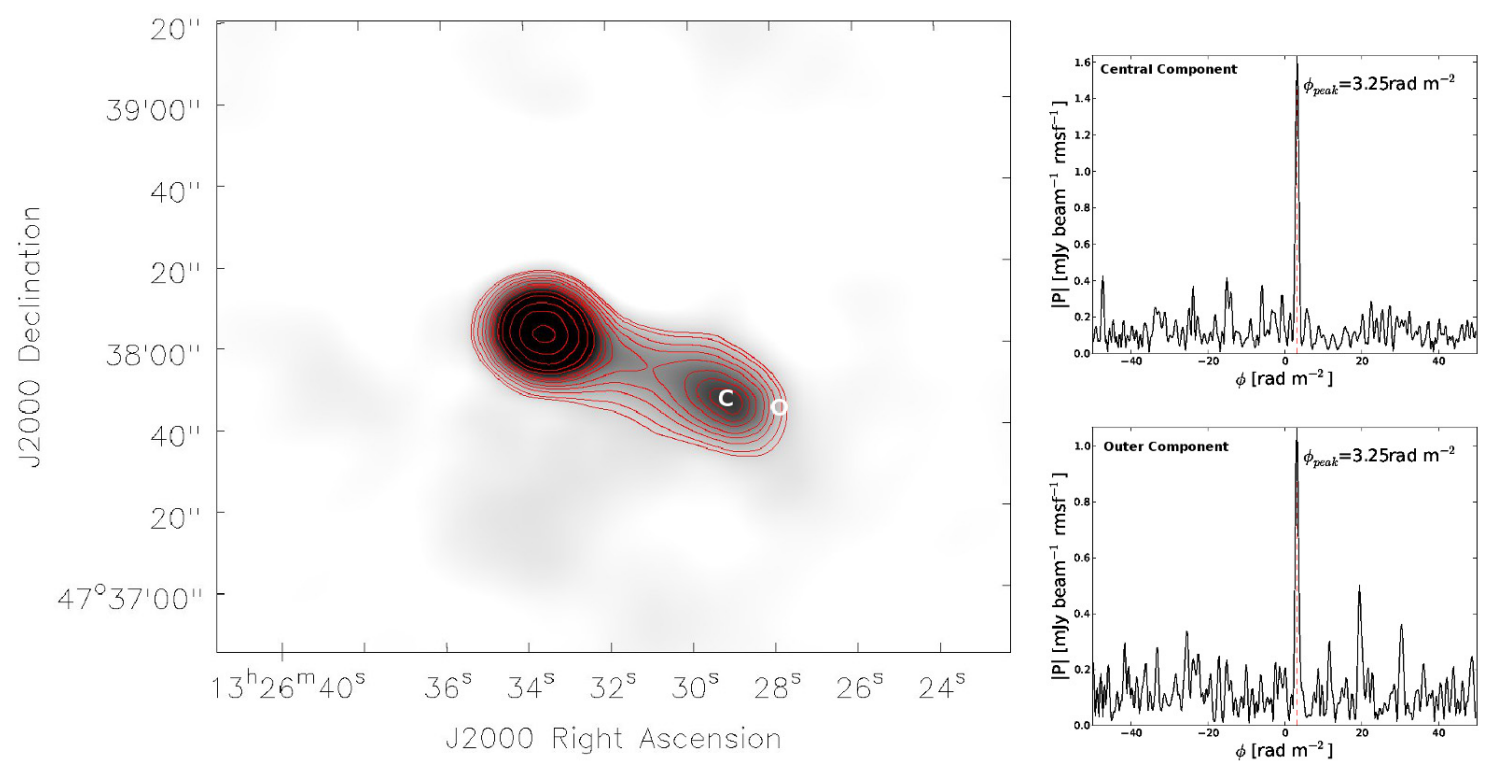

Fig. 17. Radio lobe J132626+473741 in total intensity (left) with the two marked corresponding Faraday spectra (right).
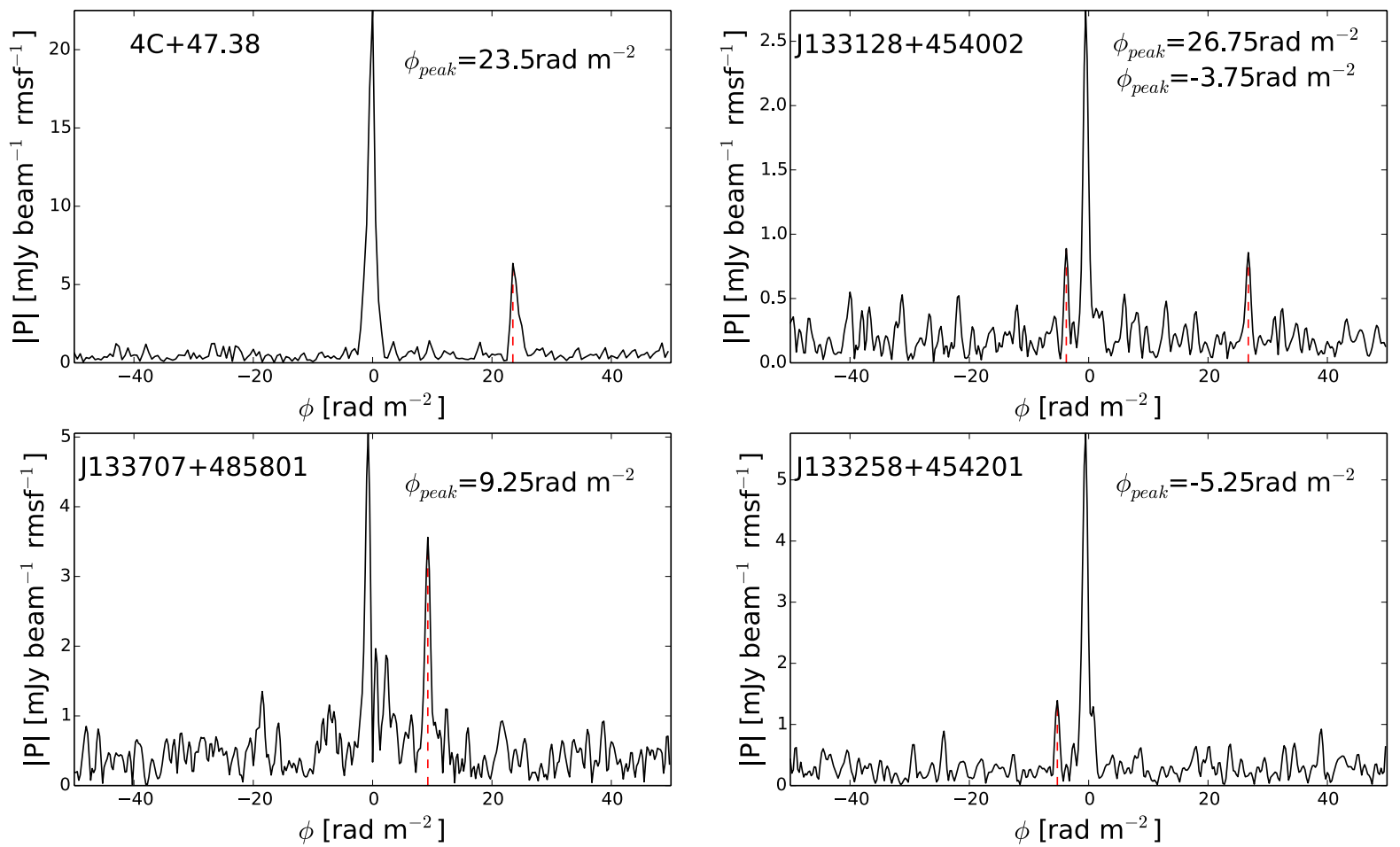

Fig. 18. Faraday Spectra of the four unresolved polarised sources detected in the M 51 field.

where $\operatorname{div}(v)$ is the adiabatic expansion term by a galactic wind, $n$ represents the bremsstrahlung loss term with $n$ being the total gas density, $w(r)$ is the radiation field representing inverse Compton loss, and $B_{\perp}^{2}$ represents the synchrotron loss term with $B_{\perp}$ being the magnetic field strength perpendicular to the line of sight. Inverse Compton loss in the galaxy's radiation field is generally smaller than synchrotron loss in galaxies (Heesen et al. 2014).

In M 51, no spectral break is observed (Fig. 4). Neglecting inverse Compton loss and adiabatic expansion and adopting an average field strength of $15 \mu \mathrm{G}$ (Fig. 9), we would need an average total gas density of more than $7 \mathrm{~cm}^{-3}$ to shift $E_{\mathrm{b}}$ beyond $10 \mathrm{GeV}$, so that no steepening occurs within the observed frequency range (Fig. 4). Such a large gas density is improbable. Typical gas densities of a few cm $\mathrm{cm}^{-3}$ lead to $E_{\mathrm{b}} \approx 2-5 \mathrm{GeV}$, hence a steepening of the synchrotron spectrum beyond $1-6 \mathrm{GHz}$ is expected.

The model by Pohl et al. (1991) did not take into account thermal absorption and thermal emission. While thermal absorption is not important for the integrated spectrum of M 51, thermal emission is significant at high frequencies and should lead to a spectral flattening. We propose that a spectral steepening occurs within the observed frequency range, but is hidden by spectral flattening due to the thermal emission.

Note that even a sharp break in the CRE energy spectrum leads to a smooth steepening of the synchrotron spectrum due 
to the broad emission spectrum of each CRE particle. Density, radiation field and magnetic field strength in galaxies vary over some range, so that no sharp break exists and the spectrum of the integrated synchrotron emission is smoothed out further. Modelling of galaxies is needed to investigate the amount of variation of synchrotron spectral index.

Indications of thermal absorption only exist in limited regions in the centre of M51. One such region lies in a complex of H II regions, namely, CCM15, 6A, 25B, 37A and 45B (Carranza et al. 1969). This supports the results of Lacki (2013) that $\mathrm{H}$ II regions are the most important locations of thermal absorption down to $10 \mathrm{MHz}$. If this is the case, we expect to detect increased thermal absorption with increasing pathlength and therefore with increasing inclination of the galaxy. Observations of edge-on galaxies with LOFAR are important to confirm this, with NGC 891 being observed already.

The spiral arms of M 51 are still observable at $151 \mathrm{MHz}$ but signs of cosmic ray diffusion of low-energy CRE are obvious. For example, the wavelet cross-correlation between $151 \mathrm{MHz}$ and $70 \mu \mathrm{m}$ is seen to have a significant correlation at scales greater than $1.45 \mathrm{kpc}$ compared to $0.72 \mathrm{kpc}$ for $1.4 \mathrm{GHz}$ and $70 \mu \mathrm{m}$. This is a measure of the propagation length of CRE that decreases with increasing frequency. We could also detect a smoother gradient in the arm-interarm contrasts at $151 \mathrm{MHz}$ compared to higher frequencies. These findings point to the fact that diffusion is the most dominant process of cosmic ray electron propagation in the star forming disk of M 51. In the Milky Way, a diffusion model with the possible inclusion of convection is the most suitable description of CR transport in the Galaxy at energies below approximately $10^{17} \mathrm{eV}$ (Strong et al. 2007). More evidence of this from observations and simulations will be presented in a future paper (Mulcahy et al., in prep.).

Other face-on galaxies with different properties, such as NGC 628 with its extended H I disk (Walter et al. 2008) need also to be examined. Several more face-on galaxies have been been or will be shortly observed with LOFAR and will shed more light on this matter. In addition, dedicated LOFAR Low Band Antenna (LBA) observations at $30-70 \mathrm{MHz}$ will be proposed where thermal absorption will become more prominent and therefore a flattening of the integrated spectrum is expected to be found.

\section{Conclusions}

We have presented the first LOFAR High Frequency Antenna (HBA) image of a nearby galaxy, namely M 51 at a central frequency of $151 \mathrm{MHz}$. LOFAR enables us for the first time to observe this galaxy at such a low-frequency with arcsec resolution and sub-milliJansky sensitivity. This observation enabled us to study aged electrons within the galaxy and therefore probe regions where the magnetic field is weak, i.e. the extended outer disk.

The main findings are summarised as follows:

1. We have been able to detect the disk of M 51 out to $16 \mathrm{kpc}$ radius. Assuming equipartition we estimate that the magnetic field strength is approximately $10 \mu \mathrm{G}$ at a radius of $10 \mathrm{kpc}$.

2. The spectrum of integrated radio emission of M51 is described well by a power law with a constant spectral index between $151 \mathrm{MHz}$ and $22.8 \mathrm{GHz}$. The lack of flattening towards low frequencies indicate that thermal absorption and ionisation losses are not important. Observations of more galaxies with LOFAR HBA \& LBA and at frequencies beyond $22 \mathrm{GHz}$ would help to verify this.
3. The lack of steepening of the integrated spectrum of M51 towards high frequencies suggests that flattening by thermal emission balances steepening by synchrotron losses of CREs. Bremsstrahlung loss dominates below the break frequency of a few $\mathrm{GHz}$, which is consistent with an average density of the total gas of a few $\mathrm{cm}^{-3}$.

4. We see evidence of free-free absorption in the central region and in H II regions in the inner arms of M51 with spectral indices flatter than -0.5 .

5. The scale length of the synchrotron emission from the outer disk $(r \geq 10 \mathrm{kpc})$ is approximately 2.6 times smaller than the scale length in the inner disk $(r \leq 10 \mathrm{kpc})$ at $151 \mathrm{MHz}$ and $1400 \mathrm{MHz}$. We assign the small scale length in the outer disk to the sharp decrease of the star formation rate and hence of the sources of CRE. Under such conditions, detecting the extreme outer disk of galaxies at low radio frequencies and in turn of magnetic fields is difficult.

6. The scale length of the synchrotron emission at $151 \mathrm{MHz}$ is approximately 1.6 times larger than that at $1400 \mathrm{MHz}$ in the inner and outer disk, because the CRE propagation length is larger at lower frequencies.

7. While the spiral arm and interarm regions are very visible in both total power and spectral index, we see significant CRE diffusion into the interarm region at $151 \mathrm{MHz}$ compared to $1.4 \mathrm{GHz}$ and conclude that diffusion is the dominant process of CRE propagation in the star forming disk of M 51.

8. The correlations between the images of radio emission at $151 \mathrm{MHz}$ and $1400 \mathrm{MHz}$ and the FIR emission at $70 \mu \mathrm{m}$ reveal breaks at scales of 1.4 and $0.7 \mathrm{kpc}$, respectively. The ratio of these break scales is also consistent with diffusive CRE propagation.

9. We estimate the average diffusion coefficient of CREs in M 51 as $D \simeq 3.3 \times 10^{27} \mathrm{~cm}^{2} \mathrm{~s}^{-1}$, smaller than the values of $D \sim(2-4) \times 10^{28} \mathrm{~cm}^{2} \mathrm{~s}^{-1}$ used by Moskalenko \& Strong (1998) for the Milky Way.

10. M 51 was not detected in polarisation at $151 \mathrm{MHz}$ due to strong Faraday depolarisation. Detection of diffuse polarisation in star forming regions of galaxies at such frequencies is difficult. Targets with significant magnetic fields but little star formation may offer chances to observe diffuse polarisation at low frequencies.

11. We detect six extragalactic sources in polarisation at $151 \mathrm{MHz}$ with a resolution of $20 \operatorname{arcsec}$ and a rms noise of $100 \mu \mathrm{Jy} / \mathrm{beam} / \mathrm{rmsf}$ in polarised intensity. In a field the size of approximately $4.1^{\circ} \times 4.1^{\circ}$, this yields a number density of polarised sources at $151 \mathrm{MHz}$ of one for every 1.7 square degrees.

12. Sources with a single lobe are far less depolarised than other sources, which is probably due to the Laing-Garrington effect. A larger sample and detailed modelling are needed to understand the depolarisation processes of radio galaxies at low frequencies. Such investigations are crucial for future low-frequency polarisation surveys like SKA-LOW, with the aim of using RM grids of background polarised sources.

Modelling of CRE propagation using the propagation and energy-loss equation is essential to understand the observational results of this paper. This is already been addressed and will be followed in a later paper (Mulcahy et al., in prep.). In addition, observations of M 51 with the LOFAR Low Band Antenna (LBA) at frequencies down to $30 \mathrm{MHz}$ would enable us to observe even further out in the galactic outer disk, and the properties of low-energy CREs, as well to determine the extent of freefree absorption in the star forming regions of M 51, in particular 
in the central starforming region. Additional face-on galaxies have been observed in LOFAR Cycle 0 and Cycle 1. This will enable us to compare galaxies with different properties, to search for diffuse polarisation in the outer galactic disks and to learn more about the strength and origin of magnetic fields in these regions.

Acknowledgements. This research was performed in the framework of the DFG Research Unit 1254 "Magnetisation of Interstellar and Intergalactic Media: The Prospects of Low-Frequency Radio Observations". LOFAR, designed and constructed by ASTRON, has facilities in several countries, that are owned by various parties (each with their own funding sources), and that are collectively operated by the International LOFAR Telescope (ILT) foundation under a joint scientific policy. We thank the anonymous referee for useful comments and Uli Klein for discussions of galaxy spectra.

\section{References}

Adebahr, B., Krause, M., Klein, U., et al. 2013, A\&A, 555, A23

Arcavi, I., Gal-Yam, A., Yaron, O., et al. 2011, ApJ, 742, L18

Baars, J. W. M., Genzel, R., Pauliny-Toth, I. I. K., \& Witzel, A. 1977, A\&A, 61, 99

Basu, A., Mitra, D., Wadadekar, Y., \& Ishwara-Chandra, C. H. 2012a, MNRAS, 419, 1136

Basu, A., Roy, S., \& Mitra, D. 2012b, ApJ, 756, 141

Beck, R. 2007, A\&A, 470, 539

Beck, R., \& Krause, M. 2005, Astron. Nachr., 326, 414

Beck, R., Brandenburg, A., Moss, D., Shukurov, A., \& Sokoloff, D. 1996, ARA\&A, 34, 155

Beck, R., Anderson, J., Heald, G., et al. 2013, Astron. Nachr., 334, 548

Bell, A. R. 1978a, MNRAS, 182, 147

Bell, A. R. 1978b, MNRAS, 182, 443

Bernardi, G., de Bruyn, A. G., Brentjens, M. A., et al. 2009, A\&A, 500, 965

Bernardi, G., Greenhill, L. J., Mitchell, D. A., et al. 2013, ApJ, 771, 105

Bhatnagar, S., Cornwell, T. J., Golap, K., \& Uson, J. M. 2008, A\&A, 487, 419

Bigiel, F., Leroy, A., Walter, F., et al. 2010, AJ, 140, 1194

Braine, J., \& Herpin, F. 2004, Nature, 432, 369

Braun, R., Heald, G., \& Beck, R. 2010, A\&A, 514, A42

Brentjens, M. A., \& de Bruyn, A. G. 2005, A\&A, 441, 1217

Carranza, G., Crillon, R., \& Monnet, G. 1969, A\&A, 1, 479

Ciardullo, R., Feldmeier, J. J., Jacoby, G. H., et al. 2002, ApJ, 577, 31

Cohen, A. S., Lane, W. M., Cotton, W. D., et al. 2007, AJ, 134, 1245

Condon, J. J., Cotton, W. D., Greisen, E. W., et al. 1998, AJ, 115, 1693

Cornwell, T. J. 2008, IEEE J. Selected Topics in Signal Processing, 2, 793

Cornwell, T. J., Golap, K., \& Bhatnagar, S. 2005, in Astronomical Data Analysis

Software and Systems XIV, eds. P. Shopbell, M. Britton, \& R. Ebert, ASP

Conf. Ser., 347, 86

Dobbs, C. L., Theis, C., Pringle, J. E., \& Bate, M. R. 2010, MNRAS, 403, 625

Dumas, G., Schinnerer, E., Tabatabaei, F. S., et al. 2011, AJ, 141, 41

Engelmann, J. J., Ferrando, P., Soutoul, A., Goret, P., \& Juliusson, E. 1990, A\&A, 233, 96

Farnes, J. S., Green, D. A., \& Kantharia, N. G. 2013 [arXiv: 1309.4646]

Finlay, C. C., Maus, S., Beggan, C. D., et al. 2010, Geophys. J. Int., 183, 1216

Fletcher, A., Beck, R., Shukurov, A., Berkhuijsen, E. M., \& Horellou, C. 2011, MNRAS, 412, 2396

Frick, P., Beck, R., Berkhuijsen, E. M., \& Patrickeyev, I. 2001, MNRAS, 327, 1145

Garrington, S. T., Leahy, J. P., Conway, R. G., \& Laing, R. A. 1988, Nature, 331, 147

Gießübel, R., Heald, G., Beck, R., \& Arshakian, T. G. 2013, A\&A, 559, A27

Gioia, I. M., \& Gregorini, L. 1980, A\&AS, 41, 329

Green, D. A. 2009, Bull. Astron. Soc. India, 37, 45

Green, D. A. 2011, Bull. Astron. Soc. India, 39, 289

Greenawalt, B., Walterbos, R. A. M., Thilker, D., \& Hoopes, C. G. 1998, ApJ, 506,135

Hales, S. E. G., Baldwin, J. E., \& Warner, P. J. 1988, MNRAS, 234, 919

Heald, G., Braun, R., \& Edmonds, R. 2009, A\&A, 503, 409

Heald, G., McKean, J., Pizzo, R., et al. 2010 [arXiv: 1008.4693]

Heesen, V., Brinks, E., Leroy, A. K., et al. 2014, AJ, 147, 103

Hinz, J. L., Rieke, G. H., Gordon, K. D., et al. 2004, ApJS, 154, 259
Horellou, C., Beck, R., Berkhuijsen, E. M., Krause, M., \& Klein, U. 1992, A\&A, 265,417

Hummel, E. 1991, A\&A, 251, 442

Iacobelli, M., Haverkorn, M., Orrú, E., et al. 2013, A\&A, 558, A72

Israel, F. P., \& Mahoney, M. J. 1990, ApJ, 352, 30

Istomin, Y. N. 2014 [arXiv: 1406.6488]

Jokipii, J. R., \& Higdon, J. C. 1979, ApJ, 228, 293

Kellermann, K. I., Pauliny-Toth, I. I. K., \& Williams, P. J. S. 1969, ApJ, 157, 1

Klein, U., \& Emerson, D. T. 1981, A\&A, 94, 29

Klein, U., Wielebinski, R., \& Beck, R. 1984, A\&A, 135, 213

Kothes, R., Fedotov, K., Foster, T. J., \& Uyanıker, B. 2006, A\&A, 457, 1081

Kulsrud, R., \& Pearce, W. P. 1969, ApJ, 156, 445

Lacki, B. C. 2013, MNRAS, 431, 3003

Lane, W. M., Cotton, W. D., van Velzen, S., et al. 2014, MNRAS, 440, 327

Lee, J. H., Hwang, N., \& Lee, M. G. 2011, ApJ, 735, 75

Lonsdale, C. J., Cappallo, R. J., Morales, M. F., et al. 2009, IEEE Proc., 97, 1497

Mathewson, D. S., van der Kruit, P. C., \& Brouw, W. N. 1972, A\&A, 17, 468

Moskalenko, I. V., \& Strong, A. W. 1998, ApJ, 493, 694

Neininger, N. 1992, A\&A, 263, 30

Offringa, A. R., van de Gronde, J. J., \& Roerdink, J. B. T. M. 2012, A\&A, 539, A95

Offringa, A. R., de Bruyn, A. G., Zaroubi, S., et al. 2013, A\&A, 549, A11

Pandey, V. N., van Zwieten, J. E., de Bruyn, A. G., \& Nijboer, R. 2009, in The Low-Frequency Radio Universe, eds. D. J. Saikia, D. A. Green, Y. Gupta, \& T. Venturi, ASP Conf. Ser., 407, 384

Patrikeev, I., Fletcher, A., Stepanov, R., et al. 2006, A\&A, 458, 441

Pohl, M., \& Schlickeiser, R. 1990, A\&A, 239, 424

Pohl, M., Schlickeiser, R., \& Hummel, E. 1991, A\&A, 250, 302

Rees, N. 1990, MNRAS, 244, 233

Rengelink, R. B., Tang, Y., de Bruyn, A. G., et al. 1997, A\&AS, 124, 259

Rieke, G. H., Young, E. T., Engelbracht, C. W., et al. 2004, ApJS, 154, 25

Roger, R. S., Costain, C. H., \& Bridle, A. H. 1973, AJ, 78, 1030

Rots, A. H., Bosma, A., van der Hulst, J. M., Athanassoula, E., \& Crane, P. C. 1990, AJ, 100, 387

Salo, H., \& Laurikainen, E. 2000, MNRAS, 319, 377

Sault, R. J., Teuben, P. J., \& Wright, M. C. H. 1995, in Astronomical Data Analysis Software and Systems IV, eds. R. A. Shaw, H. E. Payne, \& J. J. E. Hayes, ASP Conf. Ser., 77, 433

Scaife, A. M. M., \& Heald, G. H. 2012, MNRAS, 423, L30

Schinnerer, E., Meidt, S. E., Pety, J., et al. 2013, ApJ, 779, 42

Schnitzeler, D. H. F. M., Katgert, P., \& de Bruyn, A. G. 2009, A\&A, 494, 611

Schuster, K. F., Kramer, C., Hitschfeld, M., Garcia-Burillo, S., \& Mookerjea, B. 2007, A\&A, 461, 143

Scoville, N. Z., Polletta, M., Ewald, S., et al. 2001, AJ, 122, 3017

Segalovitz, A. 1977, A\&A, 54, 703

Sellwood, J. A., \& Balbus, S. A. 1999, ApJ, 511, 660

Shneider, C., Haverkorn, M., Fletcher, A., \& Shukurov, A. 2014, A\&A, in press DOI: $10.1051 / 0004-6361 / 201424192$

Silverman, J. M., Filippenko, A. V., \& Cenko, S. B. 2011, ATel, 3398, 1

Sotomayor-Beltran, C., Sobey, C., Hessels, J. W. T., et al. 2013, A\&A, 552, A58

Strong, A. W., Moskalenko, I. V., \& Ptuskin, V. S. 2007, Ann. Rev. Nucl. Part. Sci., 57, 285

Tabatabaei, F. S., Beck, R., Krause, M., et al. 2007a, A\&A, 466, 509

Tabatabaei, F. S., Beck, R., Krügel, E., et al. 2007b, A\&A, 475, 133

Tabatabaei, F. S., Krause, M., \& Beck, R. 2007c, A\&A, 472, 785

Tabatabaei, F. S., Berkhuijsen, E. M., Frick, P., Beck, R., \& Schinnerer, E. 2013, A\&A, 557, A129

Tasse, C., van der Tol, S., van Zwieten, J., van Diepen, G., \& Bhatnagar, S. 2013, A\&A, 553, A105

Taylor, A. R., Stil, J. M., \& Sunstrum, C. 2009, ApJ, 702, 1230

Theis, C., \& Spinneker, C. 2003, Ap\&SS, 284, 495

Thornley, M. D., Braine, J., \& Gardan, E. 2006, ApJ, 651, L101

Tully, R. B. 1974, ApJS, 27, 437

van der Hulst, J. M., Kennicutt, R. C., Crane, P. C., \& Rots, A. H. 1988, A\&A, 195,38

van der Tol, S., Jeffs, B. D., \& van der Veen, A.-J. 2007, IEEE Transactions on Signal Processing, 55, 4497

van Haarlem, M. P., Wise, M. W., Gunst, A. W., et al. 2013, A\&A, 556, A2

Viner, M. R., \& Erickson, W. C. 1975, AJ, 80, 931

Waldram, E. M., Yates, J. A., Riley, J. M., \& Warner, P. J. 1996, MNRAS, 282, 779

Walter, F., Brinks, E., de Blok, W. J. G., et al. 2008, AJ, 136, 2563 